\title{
A DETECTOR FOR THE STUDY OF NEUTRINO-ELECTRON SCATTERING
}

\section{The CHARM II Collaboration}

K. De Winter, J.P. Dewulf, D. Geiregat ${ }^{1}$, P. Vilain ${ }^{2}$ and G. Wilquet ${ }^{2}$ Inter-University Institute for High Energies (ULB-VUB), Brussels, Belgium

F. Bergsma, U. Binder, H. Burkard, A. Capone ${ }^{3}$, W. Flegel, B. Friend, H. Grote, A. King, C. Nieuwenhuis, H. Øverås, V. Palladino ${ }^{4}$, J. Panman, G. Piredda ${ }^{5}$, A. Seiden ${ }^{6}$, K. Winter, G. Zacek and V. Zacek CERN, Geneva, Switzerland

T. Bauche, R. Beyer, V. Blobel, F. W. Büsser, C. Foos, L. Gerland, F. Niebergall, H. Riege, J. Schütt, P. Stähelin and A. Tadsen

II. Institut für Experimentalphysik ${ }^{7}$, Universität Hamburg, Hamburg, Fed. Rep. of Germany

T. Delbar, D. Favart, G. Grégoire, E. Knoops ${ }^{1}$ and T. Mouthuy ${ }^{2}$

Université Catholique de Louvain, Louvain-la-Neuve, Belgium

P. Gorbunov, E. Grigoriev, V. Jemanov, V. Khovansky, A. Maslennikov and A. Rosanov Institute for Theoretical and Experimental Physics, Moscow, USSR

D. Braun, W. Lippich, A. Nathaniel and A. Staude

Sektion Physik der Universität ${ }^{7}$ München, München, Fed. Rep. of Germany

M. Caria ${ }^{8}$, A. Ereditato, E. Gorini, F. Grancagnolo, R. Iasevoli and P. Strolin Università e Istituto Nazionale di Fisica Nucleare (INFN), Napoli, Italy

G. Basti, D. De Pedis, E. Di Capua9 , U. Dore, A. Frenkel-Rambaldi, S. Guerra, P.F. Loverre ${ }^{10}$, R. Santacesaria and D. Zanello

Università 'La Sapienza' e Istituto Nazionale di Fisica Nucleare (INFN), Rome, Italy

(Submitted to Nuclear Instruments and Methods)

1 Inter-University Institute for Nuclear Science, Brussels, Belgium.

2 National Foundation for Scientific Research, Brussels, Belgium.

3 Facoltà di Scienze, Università di Potenza, Italy.

4 On leave of absence from INFN, Naples, Italy.

5 On leave of absence from INFN, Rome, Italy.

6 On leave of absence from Univ. of California, Santa Cruz, USA.

7 Funded by the German Ministry for Research and Technology (BMFT) under contract numbers 4HH22P and $054 \mathrm{MU} 12 \mathrm{P}$.

8 Dipartimento di Scienze Fisiche, Università di Cagliari, Italy.

9 Dipartimento di Fisica, Università di Ferrara, Italy.

10 Facoltà di Agraria, Università di Potenza, Italy. 


\begin{abstract}
The CHARM II Collaboration has built a massive, fine-grained and low-density detector for the study of muon-neutrino and muon-antineutrino scattering on electrons. The detector consists of a target calorimeter of $692 \mathrm{t}$ of glass followed by a magnetic spectrometer. The calorimeter is used to detect the different types of neutrino interactions, and to measure the energy and direction of the produced particle showers; the spectrometer permits the determination of the momentum of muons produced in charged-current reactions on nuclei. The calorimeter is composed of 441 detector planes having a total of 154,560 plastic streamer tubes with digital readout of the wires and of 73,920 cathode pick-up strips grouped in 9,240 channels with analog readout. It is further instrumented with 1,540 scintillators. A complete description of the detector and some results of its performance with cosmic-ray muons are presented.
\end{abstract}




\section{INTRODUCTION}

The Standard Model of the electroweak interactions contains, as a fundamental parameter, the weak mixing angle $\theta_{\mathrm{w}}$ defined as $\sin ^{2} \theta_{\mathrm{w}}=1-\mathrm{m}_{\mathrm{W}}^{2} / \mathrm{m}_{\mathrm{Z}}^{2}$, where $\mathrm{m}_{\mathrm{w}}$ and $\mathrm{m}_{\mathrm{Z}}$ are the masses of the intermediate vector bosons $\mathrm{W}^{ \pm}$and $\mathrm{Z}^{0}$. It relates the strength of the electromagnetic and weak forces and has to be determined experimentally. An accurate measurement of $\sin ^{2} \theta_{\mathrm{w}}$ to a precision of $\Delta \sin ^{2}$ $\theta_{\mathrm{w}}= \pm 0.005$ allows one to test the model at the level of one-loop radiative corrections. Deviations from the theoretically calculated corrections could signal the presence of new physics [1].

A particularly appealing method of measuring $\sin ^{2} \theta_{\mathrm{w}}$ is to use a purely leptonic process, where theoretical uncertainties are negligibly small. The determination of the ratio $\mathrm{R}$ of the cross-sections of neutrino-electron and antineutrino-electron scattering appears to be specially promising [2] and [3] since $R$ is related to $\sin ^{2} \theta_{\mathrm{w}}$ by

$$
\mathrm{R}=\frac{\sigma\left(\nu_{\mu} \mathrm{e}\right) / \mathrm{E}_{\nu}}{\sigma\left(\bar{\nu}_{\mu} \mathrm{e}\right) \mathrm{E}_{\bar{\nu}}}=3 \frac{1-4 \sin ^{2} \theta_{\mathrm{w}}+16 / 3 \sin ^{4} \theta_{\mathrm{w}}}{1-4 \sin ^{2} \theta_{\mathrm{w}}+16 \sin ^{4} \theta_{\mathrm{w}}}
$$

Since the cross-sections for $\nu_{\mu} \mathrm{e}$ and $\bar{\nu}_{\mu} \mathrm{e}$ scattering rise linearly with the neutrino energy and both processes are measured with the same detector, $\mathrm{R}$ can be expressed as

$$
\mathrm{R}=\frac{\mathrm{N}\left(\nu_{\mu} \mathrm{e}\right)}{\mathrm{N}\left(\bar{\nu}_{\mu} \mathrm{e}\right)} \times \frac{\int \Phi_{\bar{\nu}} \mathrm{E}_{\bar{\nu}} \mathrm{dE}_{\bar{\nu}}}{\int \Phi_{\nu} \mathrm{E}_{\nu} \mathrm{dE}_{\nu}}
$$

where $\mathrm{N}$ is the number of collected scattering events and $\Phi_{\bar{\nu}}, \Phi_{\nu}$ and $\mathrm{E}_{\bar{\nu}}, \mathrm{E}_{\nu}$ are the corresponding flux and energy of the incident neutrinos. This method is almost free of systematic errors, since the uncertainties in the knowledge of the absolute neutrino fluxes and of the detection efficiencies largely cancel in R. Only the relative normalization of the energy-weighted neutrino and antineutrino fluxes enter into the ratio. Moreover radiative corrections cancel to a large extent in $R$ (if the top quark is not too heavy [4]) and the value of $\sin ^{2} \theta_{\mathrm{w}}$ so determined can be directly related to the value determined by the boson masses $\mathrm{m}_{\mathrm{w}}$ and $\mathrm{m}_{\mathrm{Z}}$.

In order to perform a measurement of $\sin ^{2} \theta_{w}$ with a precision of \pm 0.005 , only a moderate accuracy ( $4 \%$ ) of $R$ is needed. In fact, at the current world average value of $\sin ^{2} \theta_{w}=0.23$ one gets, from the above formula, $\Delta \sin ^{2} \theta_{\mathrm{w}} \approx(1 / 8) \Delta R$.

The CHARM II Collaboration has built a detector to perform a precision measurement of the weak mixing angle by the above-described method using the wide-band neutrino beam at the CERN Super Proton Synchrotron (SPS).

The signature for neutrino-electron scattering is an isolated electromagnetic shower initiated by a single electron which is kinematically constrained to a strongly forward peaked cone of half opening angle $\Phi_{e} \leq \sqrt{2 m_{e} c^{2} / E_{e}} \approx 32 \mathrm{mrad} / \sqrt{E_{e}}$, where the electron energy $E_{e}$ is given in units of $\mathrm{GeV}$.

Most of the neutrino reactions consist of semileptonic neutrino reactions on nucleons: they outnumber purely leptonic scattering events by four orders of magnitude. Thus, efficient discrimination between electron and hadron showers must be an essential feature of the detector design.

The remaining background comes from reactions giving a shower with a large electromagnetic content in the final state. These processes are quasi-elastic scatterings of electron-neutrinos and electron-antineutrinos on nucleons, $\nu_{\mathrm{e}} \mathrm{n} \rightarrow \mathrm{pe}^{-}$and $\bar{\nu}_{\mathrm{e}} \mathrm{p} \rightarrow \mathrm{ne}^{+}$, induced by a small $\nu_{\mathrm{e}}\left(\bar{\nu}_{\mathrm{e}}\right)$ contamination of the $\nu_{\mu}\left(\bar{\nu}_{\mu}\right)$ beam and coherent and incoherent $\pi^{0}$ production on nuclei $\left({ }^{(} \bar{\nu}^{)} \mathrm{A} \rightarrow{ }^{\prime}{ }^{\prime} \pi^{0} \mathrm{~A}\right)$, induced by neutral-current interactions. The angular spread of showers generated in these processes is much broader since in the kinematical constraint the nucleon instead of the electron mass is 
involved. Restricting the analysis of the events to the range $\mathrm{E}_{\mathrm{e}} \Phi_{\mathrm{e}}^{2}<2 \mathrm{~m}_{\mathrm{e}} \mathrm{c}^{2}$ allows suppression of these background reactions. Therefore a good angular resolution is needed in order to enhance signal-to-background ratio.

Good angular resolution and efficient separation of electron and hadron showers lead to the choice of a detector of fine granularity and frequent shower sampling. Good angular resolution also requires a target material of low atomic number in order to reduce the angular spread due to multiple scattering. Fine granularity allows electromagnetic and hadronic showers to be distinguished on the basis of their different lateral profiles and energy densities. A further requirement is a large fiducial mass, since the cross-section of the purely leptonic processes is very small $\left(\sigma / \mathrm{E}_{\nu} \approx\right.$ $2 \times 10^{-42} \mathrm{~cm}^{2} / \mathrm{GeV}$ ), and the number of events needed to reach the required statistical accuracy of $\pm 2 \%$ is about 2000 for each neutrino and antineutrino beam.

To satisfy these requirements the CHARM II detector has been designed as a modular calorimeter. Each calorimeter module consists of a $4.8 \mathrm{~cm}$ thick glass plate as the neutrino target with a surface area of $3.7 \times 3.7 \mathrm{~m}^{2}$, followed by a plane of 352 plastic streamer tubes with $1 \mathrm{~cm}$ wire spacing as the active layer. Plate thickness (1/2 radiation length) and low atomic number of the target material are chosen to achieve good angular resolution for electromagnetic showers. The calorimeter is composed of 420 of these modules, the total length being $35.7 \mathrm{~m}$ and the total mass $692 \mathrm{t}$. The plastic streamer tubes are instrumented with cathode pick-up strips of $21 \mathrm{~mm}$ pitch, oriented orthogonally to the wires, for a better vertex and energy determination. Every fifth module contains, in addition, a $3 \mathrm{~cm}$ thick scintillator plane of $3 \times 3 \mathrm{~m}^{2}$ active area: for showers which originated in the glass plate preceding a scintillator plane a measurement of the energy deposited in this scintillator plane allows single-electron-induced showers to be distinguished from photon-induced showers. From these measurements the fraction of the $\nu_{\mathrm{e}}\left(\bar{\nu}_{\mathrm{e}}\right)$ contamination of the $\nu_{\mu}\left(\vec{\nu}_{\mu}\right)$ beam can be determined. In total, the calorimeter contains 154,560 digital channels of the streamer-tube system, 73,920 cathode pick-up strips read out in 9.240 analog channels, and 1,540 analog channels of the scintillator system.

To determine $\sin ^{2} \theta_{\mathrm{w}}$ from the above cross-section ratio, the energy-weighted neutrino and antineutrino flux ratio has to be known in addition to the event-rate ratio. For this purpose, the (anti-)neutrino fluxes and the energy spectra are monitored by reactions of known cross-sections. The following monitor reactions are used: the inclusive (anti-)neutrino-nucleon scattering and the $\pi^{0}$ production on nuclei. The cross-section of the quasi-elastic like reactions on the target nucleons, $\nu_{\mu} \mathrm{n} \rightarrow \mu^{-} \mathrm{p}$ and $\bar{\nu}_{\mu} \mathrm{p} \rightarrow \mu^{+} \mathrm{n}$, are almost equal at low momentum transfer for isoscalar target (equal number of protons and neutrons); corrections for the inverse $\mu$-decay reaction $\nu_{\mu} \mathrm{e} \rightarrow \mu^{-} \nu_{\mathrm{e}}$ have to be applied at very low momentum transfer. Measuring the momentum and charge of the final-state muons in a magnetic spectrometer, which directly follows the target calorimeter, allows determination of the energy spectra and of the contamination of the neutrino beam with antineutrinos and vice versa. The measured muon flux in the iron shield (see Section 4) from $\pi$ and $K$ decay in positive and negative parent beams and its comparison with the event distribution of (anti-)neutrino interactions in the detector can, in addition, be used for the flux monitoring. We are aiming at a relative flux determination with $\pm 2 \%$ accuracy.

Although the detector is optimized for the study of neutrino-electron scattering, it can also be used for the investigation of other rare neutrino processes such as coherent muon pair production, inverse $\mu$-decay and neutrino oscillations.

A detailed description of the neutrino detector is given in Sections 2 and 3, and that of the new wide-band neutrino beam in Section 4. The trigger logic is described in Section 5, the data acquisition in Section 6, and some results on the performance of the detector with cosmic-ray muons are given in Section 7. 


\section{THE NEUTRINO TARGET CALORIMETER}

To exploit the signature of small scattering angles and to suppress background components with broad angular distribution, the design of the detector has been optimized to achieve good angular resolution for electron showers. Since the determination of the direction of the shower axis is limited by multiple scattering, targets of material with low atomic number $\mathrm{Z}$ are favoured. Additional restrictions in the determination of the direction of a shower axis arise from statistical fluctuations in the sampling of showers and from the precision of the vertex measurement. These considerations suggested the choice of glass $(Z \approx 11)$ as a cheap target material, which is characterized by $\Lambda \approx 4 X_{0}$ where $\Lambda$ is the absorption length and $\mathrm{X}_{0}$ the radiation length. Electromagnetic and hadronic showers have then approximately equal lengths but differ in density and lateral profile. In hadronic reactions a wide spectrum of secondary particles is produced; these showers are characterized by low track density and a wide lateral spread, typically about $40 \mathrm{~cm}$. On the contrary, electromagnetic showers are predominantly developed through bremsstrahlung and pair production. Their transverse development is governed by the Molière radius $\varrho_{M}$ [5]; about $95 \%$ of the total energy is contained in a cylinder around the shower axis with a radius $R(95 \%) \approx 2 \varrho_{M}$, corresponding to a value of $10-11 \mathrm{~cm}$ for this calorimeter. Methods to distinguish between electromagnetic and hadronic showers are based on this characteristic difference of lateral profiles and energy densities [6].

\subsection{The modular construction of the calorimeter}

The calorimeter is composed of 420 basic modules of $3.70 \times 3.70 \mathrm{~m}^{2}$ active area, which are grouped into 21 identical units, called racks. Every one of these modules consists of a $48 \mathrm{~mm}$ thick glass target plate corresponding to one half of a radiation length, followed by a plane of 352 plastic streamer tubes [7]. The streamer tube cells are spaced out by $1 \mathrm{~cm}$, with direct readout of the wire signal in digital mode. A plane of cathode pick-up strips is fixed on the outside of the plastic envelope of the streamer tubes. It consists of $50 \mu \mathrm{m}$ thick Al-strips of $18 \mathrm{~mm}$ width, glued on one side of a 1 $\mathrm{mm}$ thick PVC sheet; the other side is covered with $50 \mu \mathrm{m}$ thick Al-foil, which acts as the ground electrode and shield. The strips are arranged in a direction orthogonal to the wires with a pitch of 21 $\mathrm{mm}$. They are used to measure in analog mode the charge induced by the streamers. This information allows the determination of the total energy of the showers and the centroid position of tracks in the projection orthogonal to the wires. There are 176 strips per plane. Figure 1 shows a picture of the CHARM II detector, details of the detector structure can be seen in Fig. 2.

Consecutive modules have wire and strip directions rotated by $90^{\circ}$. To optimize the spatial resolution of straight tracks, successive planes with the same wire direction are shifted by + or one half wire spacing. In addition, the holders, which keep the wire in position, are shifted by + or -10 to $15 \mathrm{~cm}$ to optimize the hit efficiencies for straight tracks. Only after 10 modules - corresponding to 5 radiation lengths - the spacer position sequence is repeated.

The calorimeter is also equipped with 77 hodoscope planes of scintillators. The scintillator planes are separated by 5 basic calorimeter modules. Each of these planes consists of 20 scintillators of $3 \mathrm{~cm}$ thickness and $15 \mathrm{~cm}$ width, with a total sensitive area of $3 \times 3 \mathrm{~m}^{2}$. The free area of the module, not covered by the scintillators and their light-guides, is filled up with additional material of radiation and absorption lengths identical to those of the scintillators. In this way the uniformity of the calorimeter response is maintained over the whole area.

A group of 20 basic modules and 4 planes of scintillators is followed by a plane of 320 streamer tubes with the wire direction inclined by $+7^{\circ}$ or $-7^{\circ}$ with respect to the vertical direction. These planes are not equipped with cathode pick-up strips and are used to resolve the ambiguity in the reconstruction of events with more than one track. The angle of inclination was chosen to ensure sufficient separation of multiple tracks, without imposing modifications to the basic calorimeter design. 


\subsection{The streamer-tube system and its working parameters}

The need for a target calorimeter of large mass, fine granularity, and frequent sampling suggested the use of plastic tubes for inexpensive and easy construction, operated in the limited streamer mode. The large charge of the wire signal in this mode allows the use of simple and cheap front-end electronics without preamplifiers.

The streamer tubes consist of extruded PVC profiles, each with eight U-shaped cells of $9 \times 9 \mathrm{~mm}^{2}$ inner dimensions. A $100 \mu \mathrm{m}$ diameter silver-plated $\mathrm{Cu}-\mathrm{Be}$ wire is mounted in the centre of each cell and kept in position by polyethylene wire holders spaced $47.5 \mathrm{~cm}$ apart. The 8 cells of one profile are closed by a PVC cover sheet and two of these units are mounted in a gas-tight PVC envelope forming one single chamber. The inner surfaces of each cell and its closing cover are coated with a layer of high electric resistivity. The high voltage for the streamer tubes is applied directly to the resistive layer on the cell walls, thus making it possible to read the individual wire signal without high-voltage decoupling capacitors. Each profile of a plane is connected separately to the high-voltage bus line via a $10 \mathrm{M} \Omega$ resistor, and the bus is connected to the supply via a $1 \mathrm{M} \Omega$ resistor. Details of the chamber construction and first test results can be found elsewhere [8].

Immediately after production, every chamber ran through a test cycle in order to check on the quality of the production. After a test for gas leaks, the chambers were flushed and filled with the correct gas mixture for at least five volume changes. The high voltage was applied in a certain sequence to condition the chambers. Only chambers with a dark current below $1 \mu \mathrm{A}$ at a voltage of $4550 \mathrm{~V}$ were retained for plane assembly. The test procedure is described in full detail elsewhere [9].

The gas circulating through the streamer-tube system is composed of $73 \%$ isobutane in argon with an addition of $\approx 0.5 \%$ water vapour. The adopted working voltage is $4300 \mathrm{~V}$, where a plateau of $400 \mathrm{~V}$ has been observed [8]. The high-voltage system of the streamer tubes is controlled by a small stand-alone computer, which monitors the actual values of voltage and current for every channel and allows values to be set for the current limit, ramp-up and ramp-down time, etc. The computer also writes periodically on disk the status of the system, records overcurrents and tripped channels, and switches them on again.

Up to now, after 2 years of continuous operation, only $\approx 100$ profiles out of 19,320 exhibited an abnormal value of the current $(\geq 50 \mu \mathrm{A})$. In the majority of cases a single wire was responsible for the discharge, and was then disconnected from the system. The rate of malfunctioning wires (out of a total of 154,560) has reached a stable value of 1-2 wires per month after 1 year of running time.

\subsection{The gas system}

The layout of the gas system is shown in Fig. 3; it is similar to the one used in the former CHARM detector [10]. A commercial system of thermal mass flowmeters and controllers is used to set the mixture of isobutane and argon. Its reproducibility and long-time stability is better than $\pm 0.2 \%$. The total gas flow is set at $\approx 12 \ell / \mathrm{min}$ (normalized to standard pressure and temperature) corresponding to a replacement of the total gas volume $\left(\approx 50 \mathrm{~m}^{3}\right)$ by fresh gas once every 3 days.

The gas supply system is divided into 11 independent subsystems, which allows separate operation of the different sections of the detector. To avoid trapped air pockets in the chambers owing to the low gas flow, and to homogenize outgassing impurities, a membrane pump in each subsystem recirculates the gas mixture through the streamer tubes. It recirculates the gas volume of one subunit in $4 \mathrm{~h}$. Another subsystem is used to control the fresh gas mixture. It has an additional commercial gas analyser, which compares the intensity of a specific infrared absorption line of the gas mixture with the corresponding one of a built-in test cell filled with isobutane. It should be noted that the measurements of the gas mixture with the infrared analyser have to be corrected for variations of the atmospheric pressure, whereas the thermal mass flowmeters are not affected by such small pressure changes. 
The gas flow is regulated to ensure an over-pressure in the gas subunits of about 5-8 Torr. The gas-flow system is open at the end and the used gas is released into the air. Thus the pressure in the chambers varies with the atmospheric pressure by as much as 50 Torr over long periods. To compensate the corresponding variations of the gas amplification each gas subsystem is equipped with a stabilizer. It consists of a set of 4 streamer tubes that are irradiated by a weak ${ }^{90} \mathrm{Sr}$ source. The amplified average current on these wires is kept constant by a feedback system, which regulates the high voltage on the conducting layer of the cell walls. The same high voltage is used on all the streamer tubes of this gas module. Figure 4 shows the regulated high voltage of a stabilizer as a function of the atmospheric pressure. The measurements were taken over a period of 2 months; the values are not corrected for temperature changes of the gas mixture in the stabilizers. The variation of the high voltage is $40 \mathrm{~V}$ for a change of 10 Torr in the atmospheric pressure. Without compensation this pressure variation would produce a change of $10 \%$ in the streamer charge. The r.m.s. spread of the straight-line fit is $\pm 6.5 \mathrm{~V}$; it is mainly due to temperature variations. It should be noticed that a temperature increase of $1{ }^{\circ} \mathrm{C}$ lowers the correction voltage of the stabilizers by about $10 \mathrm{~V}$.

Previous tests had shown [8] that an admixture of more than $0.4 \%$ of water vapour in the gas inhibits deposition of material on the wire. These deposits may arise during collection of large quantities of charge, for example during the high-voltage conditioning period, when some chambers draw currents of a few microamperes for several hours prior to settling down. Such deposits were found to contain large amounts of chlorine and cause an irreversible decrease of the signal. The water vapour is added by directing the incoming gas mixture over the surface of water in a small container (Fig. 3). Its admixture is monitored by another infrared analyser, calibrated for water vapour.

\subsection{The digital and analog signal processing}

\subsubsection{The digital electronic system}

The digital electronic system stores the pattern of hit wires and provides logic signals for fast trigger decisions. For every plane, 11 shaper cards, each containing the front-end electronics for 32 wires, are mounted close to the chambers. They are serially connected via a 32 bit bus to a memory card that can store the data for up to 170 events. All logical signals used to synchronize the data storage are distributed on one bus line per plane. This control bus has on one end a termination and address card that contains a hardware preset 9 bit word, representing the plane number. Figure $5 \mathrm{a}$ shows the basic circuit diagram of the shaper card; its logical interconnections are shown in Fig. 5b.

The large wire signals allow the use of a comparator as discriminator and shaper for the front-end circuit. The two diodes, on the input line from the wire, clip the signal at a level of \pm 600 $\mathrm{mV}$ and protect the downstream electronics from damage due to discharges of the wire. Any wire signal exceeding a threshold of $-30 \mathrm{mV}$ will give a TTL level of $700 \mathrm{~ns}$ length at the output of the comparator.

The comparator output is split to provide the signals for the trigger and for data storage. The trigger part of the signal is fed into an 'OR', connecting 8 wires of a chamber profile to one group signal G. The analog sum of these group signals is used for the trigger decisions (see Section 5). If minimal trigger conditions are fulfilled, a strobe signal is generated within $400 \mathrm{~ns}$.

The data part of the signal is fed to a multiplexer before the latch. This two-to-one multiplexer, together with the serial connection of the shaper cards, has two functions: a) If the SELECT status is high (see Fig. 5a) it latches the comparator output. The previous latch content is then overwritten, if a strobe signal is given by the trigger logic. b) When a higher level trigger decision initiates a transfer cycle by setting the SELECT status low, it latches data serially from the shaper cards to the memory card. During this time, no further strobe signals can be accepted. In this case, 12 clock pulses are sent 
to the shaper cards and their content, together with the content of the address word, is shifted, one after the other, into the memory card. A parity bit is generated for each of the first 11 clock pulses. The 12th word contains, besides the plane address, also these 11 parity bits plus an identifier count. In this situation, the configuration acts as a 12-word-deep shift register of 32 bits. The identifier count is generated by an overflowing 8-bit counter, which is incremented by the trigger signal. This counter acts as a synchronization for the events during the readout of the different detector parts. Whenever a synchronization error occurs, all these counters in the detector are reset.

Each shaper card contains 4 test lines, which can activate 8 channels each. In this way, special test events can be generated and read to check the system.

At the end of an accelerator cycle all memory cards are read out, event-by-event, by the data-acquisition system and are checked for consistency with the help of a parity word.

\subsubsection{The analog electronic system of the cathode pick-up strips}

The analog system measures the charge induced on the pick-up strips when ionizing particles traverse the streamer tubes. The dynamic range of the electronics has to be large enough to detect a minimum ionizing particle and to measure the charge induced by a dense electromagnetic or hadronic shower.

For reasons of cost it was not possible to equip each of the 73,920 strips with its own electronic channel. Taking advantage of the fact that electromagnetic showers have narrow lateral profiles, it was decided to combine the signals from several strips far away from each other. By combining for every plane the signals of 8 strips, spaced $46 \mathrm{~cm}$ (22 strips) apart, the number of channels was reduced to 9240 . Figure 6 shows a block diagram of the analog electronics.

To be compatible with the other electronics, the conversion time of the analog system has to be $\leq 5 \mu \mathrm{s}$. The dynamic range needed is about 1000; it is costly to obtain it in one conversion at the required speed; moreover, the data must be buffered to be read between cycles of the accelerator.

A transistor at the end of each cathode strip, biased to match the strip impedance, converts the induced voltage signal to a current at its collector. The currents from the 8 strips to be combined are connected via a matched bus line to a pulse transformer at the input of the analog circuit. This current signal is then integrated, amplified, and fed to a peak detector that holds the maximum signal from the integrator that is proportional to the input charge. The output of the peak detector is held for $1.6 \mu \mathrm{s}$ by the logic unless an event trigger arrives; in this case the signal is held until the end of the analog-to-digital converter (ADC) conversion cycle.

To obtain the required dynamic range, the output of the peak detector is digitized twice by an 8-bit flash-ADC of $1.2 \mu \mathrm{s}$ conversion time by switching the reference voltage from $1 \mathrm{~V}$ to $5 \mathrm{~V}$, giving a high and low sensitive conversion and an effective dynamic range of 1250 channels (more than 10 bits). The high-sensitivity conversion is adjusted such that the charge of the streamer of one minimum ionizing particle corresponds, on the average, to $\approx 40 \mathrm{ADC}$ counts; one ADC count is equivalent to a charge of about $0.5 \mathrm{pC}$. No significant saturation is observed for electron showers with an energy of up to $\approx 30 \mathrm{GeV}$.

After each conversion the outputs of the latched ADCs are multiplexed and transferred to static memories (arranged as 2 kwords of 8 bits) of $100 \mathrm{~ns}$ access time. The time taken per event is $3.2 \mu \mathrm{s}$, giving, with the holding time of the peak detector, a maximum conversion time of $4.8 \mu \mathrm{s}$. The memories can store the digitized analog data for up to 128 events. The readout of the memory buffers is carried out, event-by-event, between two accelerator cycles.

It should be stressed that particular care was taken in the choice of the d.c. power supplies feeding the calorimeter electronics. To minimize the electrical noise in the vicinity of very sensitive analog circuitry, switching power supplies had to be avoided. Ground connections were done without loops and gave return paths as short as possible. 
In order to test and debug the cathode strips and the readout electronics, a special pulser system was developed. A voltage pulse of adjustable height, simulating an effective charge, can be addressed to every strip by an external trigger signal. In this way, the linearity, the noise level, and the response of the electronic channels can be checked. Figure 7 shows the distribution of the strip response over the whole detector for a fixed input pulse height; the r.m.s. width is $20 \%$ of a mean pulse height, which corresponds nearly to the charge of 3 minimum ionizing particles.

\subsection{The scintillator system}

The calorimeter is instrumented with a scintillator system, which is used to measure the energy deposition of showers with a vertex in the glass plate preceding a scintillator plane. From these measurements the relative fraction of background reactions due to quasi-elastic electron-neutrino scattering and coherent $\pi^{0}$ production, as well as the flux ratio of $\left({ }^{\prime}\right)_{\mathrm{e}}^{\prime}$ and $\left({ }_{\nu}\right)_{\mu}$, can be evaluated (see Section 1).

The scintillator system consists of 1540 scintillators arranged in 77 planes of 20 scintillators each. The scintillation counters are made of polyvinyl toluene (NE114); each one is $3 \mathrm{~cm}$ thick, $15 \mathrm{~cm}$ wide, and $300 \mathrm{~cm}$ long, and is terminated by a plane mirror at the end opposite to the photomultiplier. A 12 stage photomultiplier with a 2 in. diameter window is connected to the scintillator by means of a $50 \mathrm{~cm}$ long triangular light-guide. These scintillators have already been used in the previous CHARM experiments and are described elsewhere [10,11].

Figure 8 shows the response of the scintillator system to cosmic-ray muons. The signals were collected uniformly over the whole detector and corrected for the angle of the tracks in the scintillators.

The attenuation of the pulse height along a scintillator is monitored by recording the signals of cosmic-ray muons in the time interval between accelerator cycles. After correcting these signals for the angle of incidence of the muons, the response of each scintillator is approximated by a cubic function of the distance to the light-guide; the parameters are determined for each scintillator and used in the off-line analysis to correct for attenuation losses. This calibration procedure takes into account drifts in the gain of the photomultipliers, changes in the properties of ADCs, and slow changes in the properties of the scintillators themselves. A measure of the uniformity of the scintillator response over the whole detector is the r.m.s. width of the distribution of the corrected response of the individual scintillators; this width is $\pm 5 \%$ of the mean value. A long-term stability of the response, of $\pm 2 \%$, is obtained.

\subsection{The veto system}

Together with each neutrino burst a bunch of beam-related particles, mostly muons, hit the detector. In order to screen these particles and to suppress triggers being produced by incoming muons and produced charged particles, a veto system is installed in front of the calorimeter.

The veto system is made from 4 iron plates each $8 \mathrm{~cm}$ thick interleaved with scintillator planes. Every plane consists of 26 scintillators and covers an area of $4 \times 4 \mathrm{~m}^{2}$. The efficiency of each individual counter is continuously monitored. A veto signal of $500 \mathrm{~ns}$ length is produced if at least 2 of the 4 scintillator planes are hit. The veto efficiency was found to be $>99 \%$ and the introduced dead-time is $5 \%$, about $30 \%$ of the total dead-time. 


\section{THE MUON SPECTROMETER}

In addition to the monitoring possibilities provided by the CERN SPS beam facilities, the CHARM II experiment relies on its own detector to determine neutrino and antineutrino fluxes and their respective energy spectra (see Section 1). For this purpose, muons generated in quasi-elastic neutrino-nucleon reactions are detected, and the primary neutrino spectra are obtained by measuring the muon spectra. Since the muons produced in quasi-elastic reactions are emitted at small angles with respect to the neutrino beam, a spectrometer situated immediately behind the target calorimeter and covering its area provides the necessary acceptance*

The muon spectrometer consists of 6 circular magnetized iron modules and 9 hexagonal drift chambers. Drift chambers and magnet modules are arranged alternately, with one additional drift-chamber module preceding and one following the set-up. Figure 2 shows some details of the arrangement. Each magnet module is constructed of iron plates, with a total thickness $50 \mathrm{~cm}$, interleaved with 4 planes of scintillation counters. Each of the drift-chamber modules is equipped with three planes of sense wires, oriented at $0^{\circ},+60^{\circ}$, and $-60^{\circ}$ with respect to the horizontal.

Signals from the scintillation counters are used to trigger on quasi-elastic neutrino events, requiring that a muon traverses at least two magnet modules. The hits in the drift chambers are used in the off-line analysis to reconstruct the track and the momentum of traversing muons. The momentum resolution is essentially limited by multiple scattering of the muons in the iron and amounts to $\pm 14 \%$ for a muon of $20 \mathrm{GeV} / \mathrm{c}$ momentum.

\section{THE NEUTRINO BEAM}

Because of the very small cross-sections of neutrino-electron scattering, the use of high-intensity neutrino beams is required. The CHARM II detector is exposed to the wide-band neutrino beam of the SPS at CERN. Details of the SPS neutrino beam are given elsewhere [13]. A number of modifications have been applied to the beam set-up to achieve the highest possible muon-neutrino flux in the energy range favourable for detecting neutrino-electron scattering events with the CHARM II detector. The extraction of the proton beam has been modified in order to get two spills at the full SPS energy. In addition, a new set of focusing elements is used which is optimized for a high yield of mesons in the favoured energy range.

A schematic layout of the beam line is shown in Fig. 9. The SPS accelerates protons to $450 \mathrm{GeV}$ with a cycle of $14.4 \mathrm{~s}$. Protons are extracted at the maximum SPS energy to the neutrino target in two $6 \mathrm{~ms}$ long spills, spaced in time by $2.8 \mathrm{~s}$. The large spill length reduces the thermic stresses in the target and diminishes the dead-time of the CHARM II detector. Typical intensities are $1.1 \times 10^{13}$ protons on target for the first spill and $0.7 \times 10^{13}$ protons on target for the second spill.

The target consists of 11 beryllium rods of $3 \mathrm{~mm}$ diameter and $10 \mathrm{~cm}$ length, spread at equal distances from each other along the beam line over a range of $2 \mathrm{~m}$, corresponding to 3 interaction lengths. The small diameter and the segmentation minimize the reabsorption of secondary particles - mostly pions and kaons - produced in the primary proton-beryllium collisions. Hadrons emerging from the target under large angles are absorbed in a collimator of $8 \mathrm{mrad}$ aperture.

Forward-going hadrons are sign-selected and focused in two magnetic lenses called 'horn' and 'reflector', respectively, whose principle was invented by S. van der Meer. These are coaxial conductors, which produce an axial symmetric magnetic field during the time of the proton extraction. For this purpose LC networks shape the horn and reflector currents to nearly constant

*) The muon spectrometer with its electronics has kindly been lent to us by the CDHS Collaboration. It is described in detail elsewhere [12]. We summarize only the main features here. 
values during the $6 \mathrm{~ms}$ extraction time. Over a wide range of momenta and impact angles, hadrons of one sign are bent towards an evacuated decay tunnel while hadrons of the opposite sign are swept out. The shape of the inner conductor of the horn was designed to achieve the highest focusing efficiency for mesons of about $70 \mathrm{GeV}$ that are the parents of neutrinos with energy up to $40 \mathrm{GeV}$.

In the evacuated tunnel (residual air pressure $\approx 1 \mathrm{mbar}$ ) a fraction of the hadrons decay. All surviving hadrons are absorbed within the first few metres of the following iron shield. The muons, produced together with the neutrinos, are swept out by a toroidal magnet or ranged out in the iron and earth shields. An additional iron shield of $12 \mathrm{~m}$ was installed in front of the detector to reduce the higher number of penetrating muons due to the increase of the primary proton energy to $450 \mathrm{GeV}$.

Selecting positive hadrons results in a $\nu_{\mu}$ beam and negative hadrons in a $\bar{\nu}_{\mu}$ beam. The $\nu_{\mu}\left(\bar{\nu}_{\mu}\right)$ beam has a component of $\bar{\nu}_{\mu}\left(\nu_{\mu}\right)$ due to wrong-sign hadrons entering the decay tunnel. In addition, the beams contain electron-neutrino components of the order of $1 \%$ from $\mathrm{K}_{\mathrm{e} 3}$ decays. The opposite helicity contamination in the neutrino beam is about $7 \%$. In the antineutrino beam this contamination is higher because positive hadrons are produced more copiously and with a higher mean energy. To reduce this effect, an additional beryllium rod of $118 \mathrm{~cm}$ length and $16 \mathrm{~mm}$ diameter is placed in front of the horn during antineutrino periods. This 'stopper' absorbs a sizeable amount of high-energy positive mesons produced at small angles which would not be deflected by the focusing elements; the resulting contamination in the antineutrino beam is reduced to approximately $13 \%$.

The flux in the CHARM II detector amounts to about $1.3 \times 10^{10}$ neutrinos per $\mathrm{m}^{2}$ for $10^{13}$ protons on target in the case of the neutrino beam and is about $50 \%$ lower in the case of the antineutrino beam. The average energy is $24 \mathrm{GeV}$ for the neutrino beam and about $20 \mathrm{GeV}$ for the antineutrino beam.

Several monitoring detectors are installed along the beam line. The intensity of the primary proton beam is measured by a beam-current transformer. The beam size and steering on the target is continuously monitored by split foils. At regular intervals intensity scans are done with a wire that is moved across the beam vertically and horizontally in steps of $0.25 \mathrm{~mm}$. The multiplicity of secondary hadrons produced in the target is measured by secondary emission monitors before and after the target. A system of solid-state detectors, installed in gaps at different depths in the iron shield and at several distances from the beam axis, is used to measure the muon-flux profile, which is closely correlated with the neutrino flux.

The time structure of the muon flux is measured by small scintillation counters located in gaps at the end of the iron shield. Folding the muon intensity with the busy signals of the CHARM II detector determines the loss of neutrino events due to dead-time. The dead-time is typically $15 \%-20 \%$ in the neutrino beam and about $10 \%-15 \%$ in the antineutrino beam.

\section{THE TRIGGER LOGIC}

\subsection{General layout of the trigger system}

The trigger is designed to take fast decisions with high selectivity, while remaining versatile. The main sources of trigger decisions are the signals provided by the digital readout of the streamer tubes. Special effort was made in order to have high trigger efficiency for low shower energy.

In a normal neutrino run with double extraction of the proton beam from the SPS and $14.4 \mathrm{~s}$ accelerator cycle (see Section 4), each of the two neutrino spills is covered in time by a $7.5 \mathrm{~ms}$ wide gate, called the 'physics' gate. During the two spills a total of 10 different types of 'physics' triggers is recorded. After the second spill, cosmics as well as pedestal and detector test triggers are enabled. In tuning the trigger requirements, limitations are set by the available buffer size (128 events per accelerator cycle for the analog system) and the demand of tolerable dead-time losses. During the 
physics gates of an accelerator cycle for $1.5 \times 10^{13}$ protons on target there are about 500 neutrino interactions, 300 tracks from incoming beam related muons, and 300 tracks from cosmic-ray muons.

The task of the trigger is to select only those events that are relevant to the aims of the experiment. The various types of selection are based on characteristics of the event, like the presence of signals in the veto system or in the muon spectrometer, the location of the event vertex, the recognition of long muon tracks in the calorimeter and estimates of limits on the shower width and length. About $80(60)$ triggers are recorded in the physics gates during a $\nu(\bar{\nu})$ wide-band beam exposure with $1.5 \times 10^{13}$ protons on target. Out of the total dead-time of $15 \%-20 \%$ for the neutrino beam, $4.5 \%$ is due to cosmic-ray muons; the rest is equally split into contributions from the veto system, event readout, and trigger-decision time.

The trigger system, similarly to the detector, is subdivided into 21 racks, each consisting of 2 half-racks of 10 planes. A plane is subdivided into 44 groups of 8 wires. A group generates a signal $\mathrm{G}$ if at least one wire is hit (see Fig. 5a). All G-signals of a plane are combined in an analog sum. From this, further logic signals are derived by requiring $\geq 1, \geq 2, \geq 3$, and $\geq 5$ groups. These signals are further combined on a rack or half-rack basis and constitute the trigger-decision input signals.

The trigger decision is made in three steps. A first level is provided by a so-called strobe signal, issued if at least 4 planes are hit in at least one half-rack, including overlaps from the preceding half-rack. The strobe signal serves to latch the wire hit pattern of the digital tube readout (see subsection 2.4.1) and provides the timing signal to start the pretrigger, which makes a fast decision on the event length by requiring more than 10 planes hit in any two subsequent racks. If this condition is fulfilled, the main trigger decision is initiated. Clusters of programmable logic units process the decision input signals together with other signals that control downscale factors and flag the status of the accelerator cycle. The decision is completed after $1 \mu \mathrm{s}$, and various trigger bits are issued to logic matrices that steer the readout of the individual detector components. After $5 \mu$ s the detector is ready to accept a new event.

\subsection{The physics and cosmic-ray muon trigger}

A detailed description of the main trigger functions is given below; all rates are quoted for $1.5 \times 10^{13}$ protons on target.

The electron trigger defines loose conditions on electromagnetic showers. A minimum event length is required, where at least 14 planes are hit in two neighbouring half-racks. An upper bound on the event length is set by admitting no more than 7 half-racks with at least 4 planes hit. In addition, an acceptance window for the shower width is defined. A lower threshold is set by requiring at least 2 wire groups to be hit in at least 3 planes of at least one half-rack. Two planes from the preceding half-rack are included as overlap. In each projection, one or more hits must be found. Broad neutral-current events are rejected if at least 5 wire groups are hit in at least 9 planes of at least one half-rack. Again two planes from the preceding half-rack are included in the overlap. The trigger efficiency for electromagnetic showers is tuned to be close to $100 \%$ between 3 and $40 \mathrm{GeV}$, as verified in a separate test run with electron beams, see Fig. 10. Inefficiencies at low energies arise from events that do not meet the minimum length and width criteria. At high energies, the inefficiency comes from the veto requirement for neutral-current events. The effective energy threshold is around $2 \mathrm{GeV}$. For all physics triggers, an anticoincidence with the veto system is required to suppress unwanted beam-associated muons (see subsection 2.6). Typical trigger rates are 50 and 35 events per cycle during $\nu$ and $\bar{\nu}$ runs, respectively. Of these events, about 25 are due to short cosmic-ray muons, which cannot be distinguished from electron showers because no information on the track angle with respect to the beam direction is available. 
The quasi-elastic trigger accepts events containing a muon that penetrates the spectrometer and hits at least 8 scintillator planes. Inelastic charged-current events are rejected by discriminating against shower activity at the vertex. The rejection corresponds to an effective shower energy threshold of $5 \mathrm{GeV}$. The efficiency of the energy threshold is larger than $98 \%$ for genuine quasi-elastic events. A small correction has to be applied in the analysis for the loss of events with an energetic bremsstrahlung shower developed by the muon track. The acceptance for quasi-elastic events is entirely determined by geometry. The trigger rate is about 10 events per cycle.

The charged-current trigger is designed to accept events within a region of the detector where the muon reconstruction efficiency is optimal. The size of this fiducial volume is chosen to minimize acceptance corrections. The trigger requires a muon in the muon spectrometer and restricts event vertices to a fiducial volume made up of the downstream half of rack 15 and racks 16, 17, 18 only. The calorimeter scintillators introduce a lateral fiducial volume cut by requiring one hit in two planes out of a group of 16 consecutive planes; it largely suppresses beam-associated muons entering the side faces of the calorimeter. About 6 (3) charged-current triggers per cycle are recorded during $\nu(\bar{\nu})$ runs, respectively.

The trident trigger is tuned to select events with two muons leaving the vertex. The two-track sensitivity of this trigger allows a spatial resolution of muon pairs as close as $10 \mathrm{~cm}$ to each other in the calorimeter. The observed trigger rate of 3 events per cycle is mostly due to accidental coincidences (double events). The physics interest of this trigger is the observation of coherent muon pair production $\left(\vec{\nu}_{\mu}{ }_{\mu}+\mathrm{A} \rightarrow{ }^{(} \bar{\nu}_{\mu}{ }_{\mu}+\mu^{+}+\mu^{-}+\mathrm{A}\right.$, so-called 'tridents' (three outgoing leptons).

The dimuon trigger asks for two muon tracks in the spectrometer. It is used for dimuon studies and to monitor the efficiency of the trident trigger for those events that have two muons entering the magnet. The rate is 2 events per cycle.

The cosmics trigger is tuned to select single muons, suitable for calibration of the various detector components. Clean tracks without bremsstrahlung showers are required and nearly horizontal cosmic-ray muons are selected, which penetrate at least 12 half-racks of the calorimeter. Usually 10 events of this type are recorded per accelerator cycle. The cosmic trigger is complemented by a veto cosmics trigger and a magnet cosmics trigger. These triggers compensate longitudinal edge effects by enhancing the trigger rate in the first and last parts of the detector.

The sample trigger requires only a lower cut on the event length of at least 10 planes hit in any one rack. It therefore selects events with minimum bias and is used to check and monitor the performance of the various trigger types. To achieve a tolerable count-rate of 7 events per cycle, it is scaled down by a factor of 60 . The rate of events in the fiducial volume is used for monitoring the neutrino flux.

A special alignment trigger selects muon tracks traversing the entire detector. Data with this trigger are taken in periodic intervals, in order to check the correct position of all sensitive elements of the calorimeter and the muon spectrometer, and their alignment with respect to the nominal axis of the neutrino beam. A precision of about $1 \mathrm{~mm}$ is reached for the streamer tubes, the pick-up strips, and the drift chambers, and of about $3 \mathrm{~mm}$ for the scintillator positions in the calorimeter.

\section{THE DATA ACQUISITION AND ON-LINE MONITORING}

The requirements concerning the data-acquisition system are determined by the duty cycle of the accelerator, the amount of data expected for each cycle, the decision to do on-line data compression by software, and the wish to use commercially available hardware when possible.

During the two neutrino spills events are stored in local memories, which can hold 40 events for the muon spectrometer, 50 events for the scintillator system, 128 events for the analog system of the cathode pick-up strips, and 170 events for the digital system of the streamer tubes. The buffer size of the muon spectrometer is sufficient to record all events triggered by a muon entering the 
spectrometer. The limitation of the scintillator system requires the $\mathrm{CC}$ trigger to be active in a restricted fiducial volume.

An ND-100/ND-500 complex from Norsk Data is used as the on-line computer system. The ND-100 computer is dedicated to data acquisition and controlling the detector, while the ND-500 computer performs monitoring tasks. The communication with the detector hardware is done through a CAMAC system.

Integrated in the CAMAC system in the form of intelligent crate controllers are three fast processors, so-called CAMAC boosters (LeCroy $4804 \mathrm{CAB} C \mathrm{CG}$ ), which carry out the data transfer from the local detector memories to the data-acquisition computer. They perform the readout, data compression, software cosmic-ray rejection, event display, and basic checks of data consistency. To optimize the performance of the system the three fast processors and the computer run in parallel, synchronized by a program running on the ND-100.

The CAMAC booster performing the data readout of the digital tubes is also used for a fast identification of background events. The main background contribution for electron trigger events (see Section 5) comes from cosmic-ray muons; for quasi-elastic trigger events it comes from beam-related muons entering the target calorimeter from the side. A large fraction of these events are rejected by a simple algorithm.

The data volume to be treated for each event is about $50 \mathrm{kbyte}$, which typically is compressed to 5 kbyte. The readout time is on an average $70 \mathrm{~ms}$ per event; the full buffer content can be written to tape in less than $9 \mathrm{~s}$ before the next accelerator cycle.

An ND-10S computer is used to receive data on beam performance from the neutrino-beam control and SPS computers, and to perform the acquisition of data on the muon flux in the iron shield. This information is sent to the main acquisition system for each cycle before the detector data are read out.

The performance of the detector and the neutrino beam is continuously monitored using the incoming data to check the functioning of the different detector elements, the quality and timing of the beam ejection, the muon flux in the iron shield, the trigger rates, the dead-time of the detector, and to update periodically run information. The accumulated histograms and tables can be displayed and printed without interfering with the data taking.

The total number of low-voltage supply channels for the front-end analog and digital electronics plus the number of NIM and CAMAC crates amounts to about 1800. All channels are monitored by a stand-alone computer that compares the measured values with those of a look-up table. An alarm is issued if a significant discrepancy is found.

\section{PERFORMANCE OF THE DETECTOR}

In the following section the response of the detector to typical neutrino interactions is shown and results obtained with cosmic-ray muons concerning the performance of the analog system of the pick-up strips are discussed. Further information on the performance of the streamer tubes and their digital readout with cosmic-ray muons is given elsewhere $[8,14]$. Results of test measurements with electron and pion beams will be published separately.

The larger part of the neutrino reactions consists of semileptonic neutrino-nucleon scattering events, as pointed out in Section 1. Figure 11a shows a hit-display of a semileptonic CC reaction as seen in the digital system of the CHARM II detector. It is characterized by a broad (hadronic) shower together with a muon track that penetrates the subsequent calorimeter part and the magnetic spectrometer where its charge and momentum are measured. Figure $11 \mathrm{~b}$ displays a $\left(\nu_{\mu} \mathrm{e}\right)$ candidate event. It shows a narrow (electromagnetic) shower, created within a small angle around the beam axis. The difference in density of hadronic and electromagnetic showers is obvious from the two figures and is used to distinguish between the two shower types. 
The response of the 73,290 strips was measured by sampling a large number of cosmic minimum ionizing particles. No corrections were applied for different track-length of the particles in the gas of the streamer tubes. The measurements show that for a hit centred on a strip, the two neighbouring strips each contain about $30 \%$ of the charge of the central strip. The sum of the charge of these 3 strips above a threshold is defined as the cluster charge. Figure 12 displays the measured cluster charge for cosmic-ray muons over the whole detector. The mean cluster charge is about $30 \mathrm{pC}$; its FWHM is $50 \%$ with a tail to larger pulse-heights coming from events with more than one streamer on a strip.

In order to describe the uniformity of the strip response over the whole detector the mean pulse height of the central strip of a cluster was computed. The r.m.s. spread of the mean pulse height from strip to strip is $\pm 20 \%$, as shown in Fig. 13. It is due to differences in the gas amplification in the capacities of the pick-up electrodes and to the different sensitivities of the amplifiers. The effect of the different electronic components is monitored separately by the pulser system; its contribution is about half of the observed r.m.s. spread.

Measurements of the stability of the cluster charge for cosmic-ray muons are given in Fig. 14. The distribution of the mean cluster charge is shown for different runs during a period of 2 weeks. Its r.m.s. spread is $2 \%$ of the mean charge, whereas without stabilizers the charge would vary up to $25 \%$ depending on the variations of the atmospheric pressure.

The charge information from the pick-up strips is used to measure the energy of showers and to determine the position of tracks in the projection orthogonal to the wires. For on-line checks during running periods a simple algorithm, using the digital information of the streamer tubes, is applied to reconstruct tracks. If the position of the track predicted from the digital information coincides with the strip containing the largest charge, or with the two neighbouring strips, the plane is declared efficient for this track. Figure 15 shows the on-line distribution of this efficiency for all planes of the calorimeter. The mean efficiency is $\approx 94 \%$; the inefficiency is mainly due to residual noise.

The noise in the analog channels of a given plane is estimated by comparing the cluster charge of a cosmic-ray track with the charge of a cluster far away from the track. Figure 16 shows the distribution of the ratio of this noise charge to the cluster charge of reconstructed tracks as measured for all calorimeter planes; its average value is $6 \%$.

\section{Acknowledgement}

The realization of this detector was possible thanks to the skill and dedication of the many people who contributed to various aspects. We should like to express our gratitude and deep appreciation to our numerous collaborators from the different participating institutes. In particular, we wish to thank R. Brocard, E. Brunetto, A. Duchène, W. D. Glessing and G. Lebée (CERN) as well as A. Utenkov (Moscow) for their help during the construction and installation phase of the detector; P. Parascandolo (Naples) for his contribution to the development of the digital electronics; J.C. Berset (CERN) for the design of the peak detector; L. Bonnet, B. de Callatay, J. M. Delforge and $M$. Jacques (Louvain) for their help and competence in designing and improving the analog electronic circuitry; A. Tusi (Rome) for repair and installation of ADCs and photomultiplier electronics; H. Herbert, P. Klemm, G. Miller and H. Willaredt (Munich) for their help in assembling the streamer-tube planes, installing the spectrometer, and for their continuous care of the gas system.

The CDHS Collaboration have kindly allowed us to use part of their equipment and electronics for the muon spectrometer; the help of the Département de Physique des Particules Elémentaires (CEN-Saclay) during the installation of the drift chambers is warmly acknowledged.

We gratefully acknowledge financial support from the Inter-University Institute for Nuclear Sciences (Belgium), CERN (Geneva), the Bundesministerium für Forschung und Technologie (Federal Republic of Germany), the Institute for Theoretical and Experimental Physics (Moscow), and the Istituto Nazionale di Fisica Nucleare (Italy). 


\section{REFERENCES}

[1] W.J. Marciano, Proc. Int. Lepton and Photon Symposium, Cornell, 1983.

A. Sirlin, Phys. Rev. D 29 (1984) 89.

W.J. Marciano and A. Sirlin, Phys. Rev. D 29 (1984) 945.

[2] CHARM II Collaboration, Proposal to study neutrino-electron scattering at the SPS, CERN/SPSC/83-24 (28 April 1983), Addendum to the proposal to study neutrino-electron scattering at the SPS, CERN/SPSC/83-37 (31 May 1983).

[3] J. Dorenbosch et al., CHARM Collaboration, Experimental results on neutrino-electron scattering, to be published in Z. Phys. (1988).

[4] J. Ellis and G. Fogli, preprint CERN-TH/88-5107 (1988).

[5] B. Rossi, High Energy Particles (Prentice-Hall, New York, 1952).

[6] K. De Winter et al., CHARM II Collaboration, An electron-hadron separator for digital sampling calorimeters, to be published in Nucl. Instrum. Methods (1988).

[7] G. Battistoni et al., Nucl. Instrum. Methods 176 (1980) 297.

E. Iarocci, Nucl. Instrum. Methods 217 (1983) 30.

[8] J.P. DeWulf et al., CHARM II Collaboration, Nucl. Instrum. Methods A252 (1986) 443 and A263 (1988) 109.

[9] M. Caria et al., Nucl. Instrum. Methods A260 (1987) 368.

[10] A.N. Diddens et al., CHARM Collaboration, Nucl. Instrum. Methods 176 (1980) 189.

[11] J. Dorenbosch et al., CHARM Collaboration, Nucl. Instrum. Methods A253 (1987) 203.

[12] M. Holder et al., Nucl. Instrum. Methods 148 (1978) 235.

[13] E.H.M. Heijne, CERN 83-06 (July 1983) (unpublished).

[14] K. De Winter et al., CHARM II Collaboration, Experimental results obtained from a low- $Z$ fine-grained electromagnetic calorimeter, to be published in Nucl. Instrum. Methods (1988). 


\section{Figure captions}

Fig. 1 Picture of the CHARM II detector. The neutrino beam enters at the lower right corner.

Fig. 2 A schematic view of the CHARM II detector.

Fig. 3 The gas system for the streamer tubes of the calorimeter.

Fig. 4 Variation of the stabilizer voltage with atmospheric pressure.

Fig. 5 Digital readout of the streamer tubes and planes; a) the front-end electronics of a shaper card, b) its logical interconnections.

Fig. 6 Block diagram of the analog electronics for the pick-up strips.

Fig. 7 Dispersion of the strip response for all planes of the calorimeter from a pulser run with fixed pulse height.

Fig. 8 Scintillator response for minimum ionizing particles (cosmic-ray muons).

Fig. 9 Schematic layout of the wide-band neutrino beam.

Fig. 10 Trigger efficiency for electromagnetic showers as a function of electron energy.

Fig. 11 Event displays of neutrino interactions in the CHARM II detector. The neutrino beam enters from the left. Each point in the calorimeter or spectrometer represents a wire hit; a short line in the spectrometer represents a scintillator hit. $\mathrm{Y}$ and $\mathrm{Z}$ are the vertical and horizontal projections of the streamer tubes in the calorimeter and the scintillators in the spectrometer; $\mathrm{Z}, \mathrm{U}$ and $\mathrm{W}$ are the 3 projections of the spectrometer drift chambers.

a) A charged-current neutrino interaction with a single muon traversing the subsequent calorimeter and the spectrometer.

b) A candidate event of a neutrino electron reaction.

Fig. 12 The distribution of the cluster charge (sum of 3 strips) for minimum ionizing particles (cosmic-ray muons).

Fig. 13 The distribution of the mean response of individual strips for minimum ionizing particles (cosmic-ray muons).

Fig. 14 Distribution of the cluster charge for data runs taken during a run period of two weeks.

Fig. 15 Distribution of the efficiency of the pick-up strips to reconstruct tracks.

Fig. 16 Distribution of electric noise on the pick-up strips. 


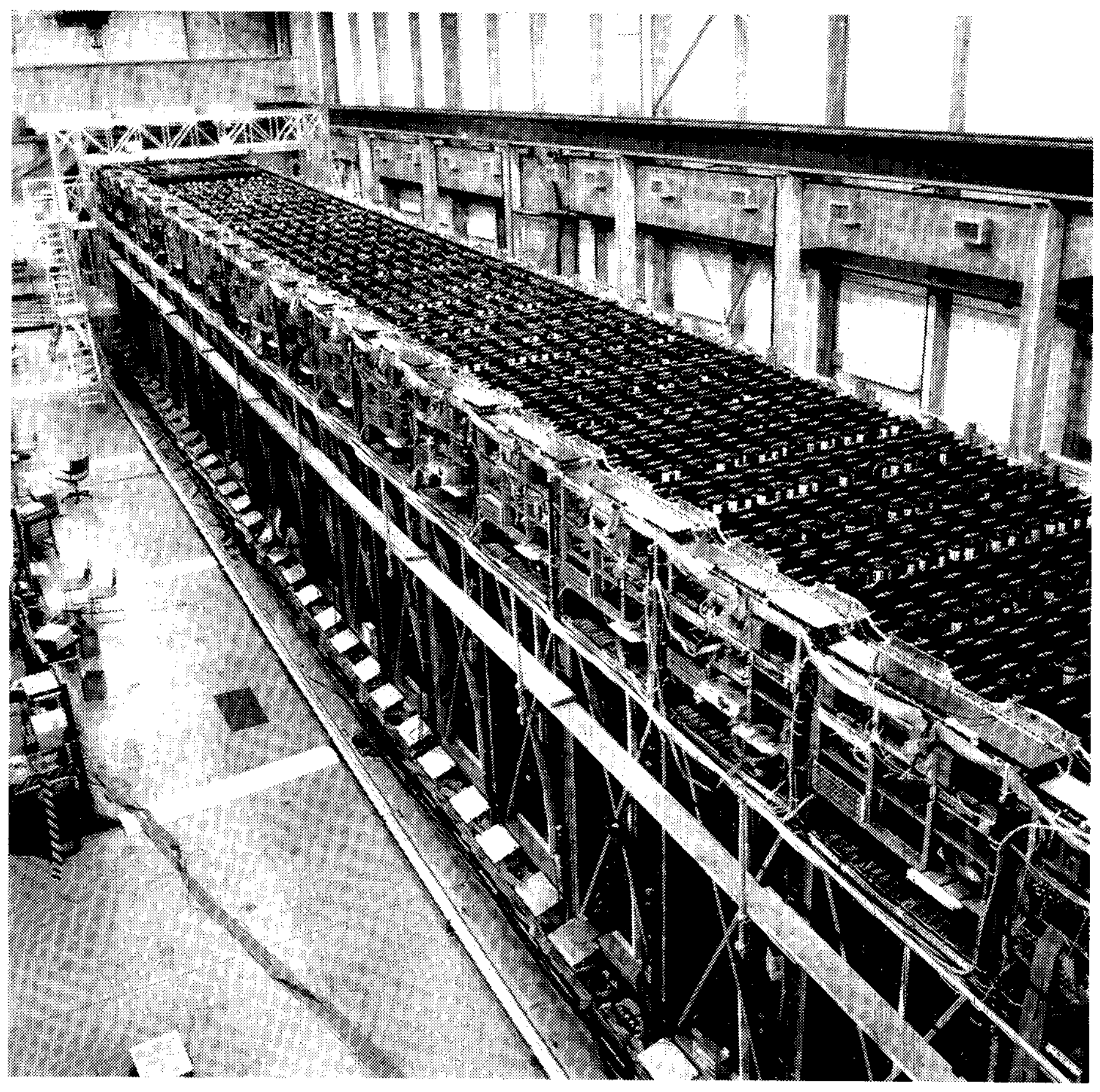

Fig. 1 


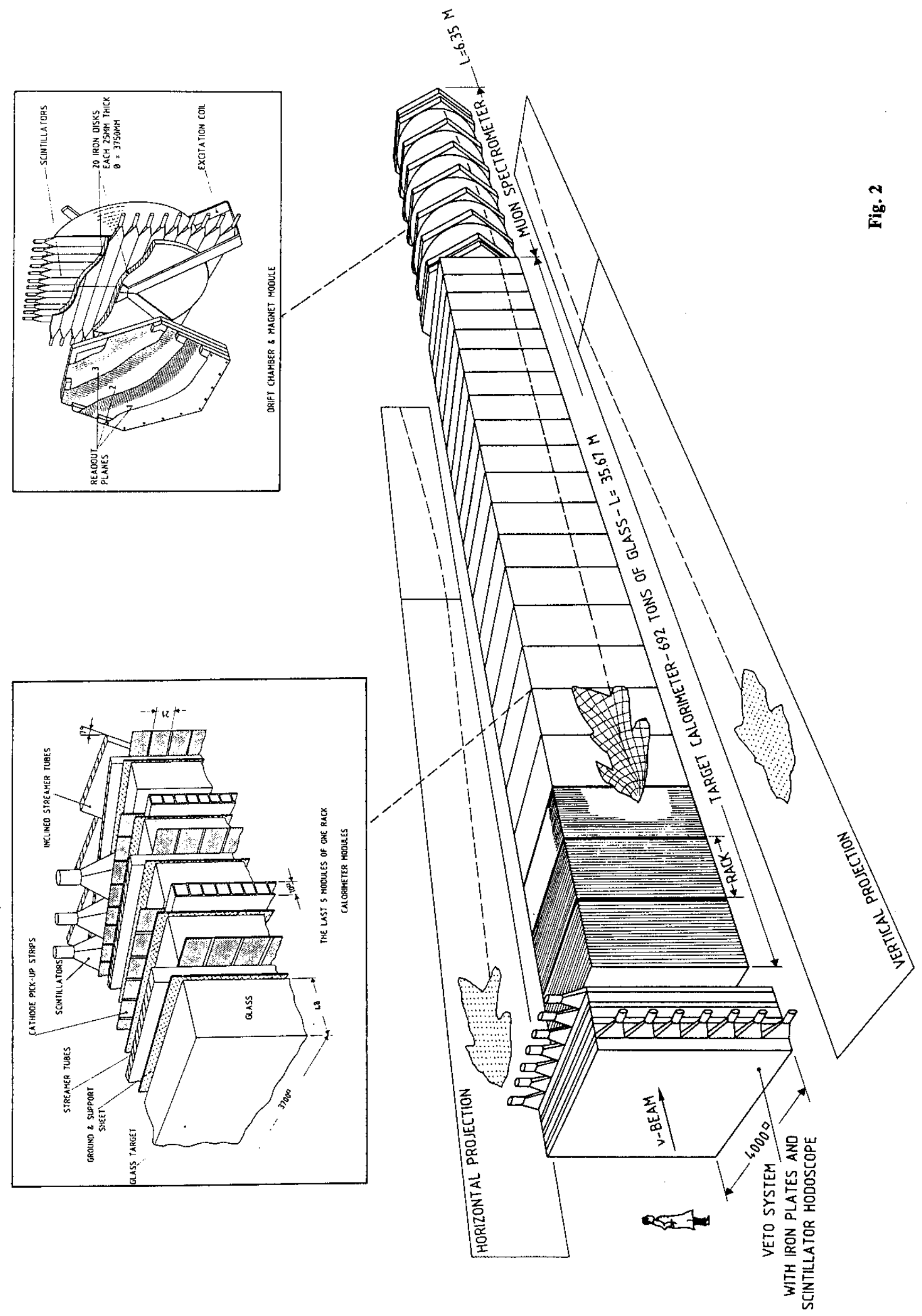




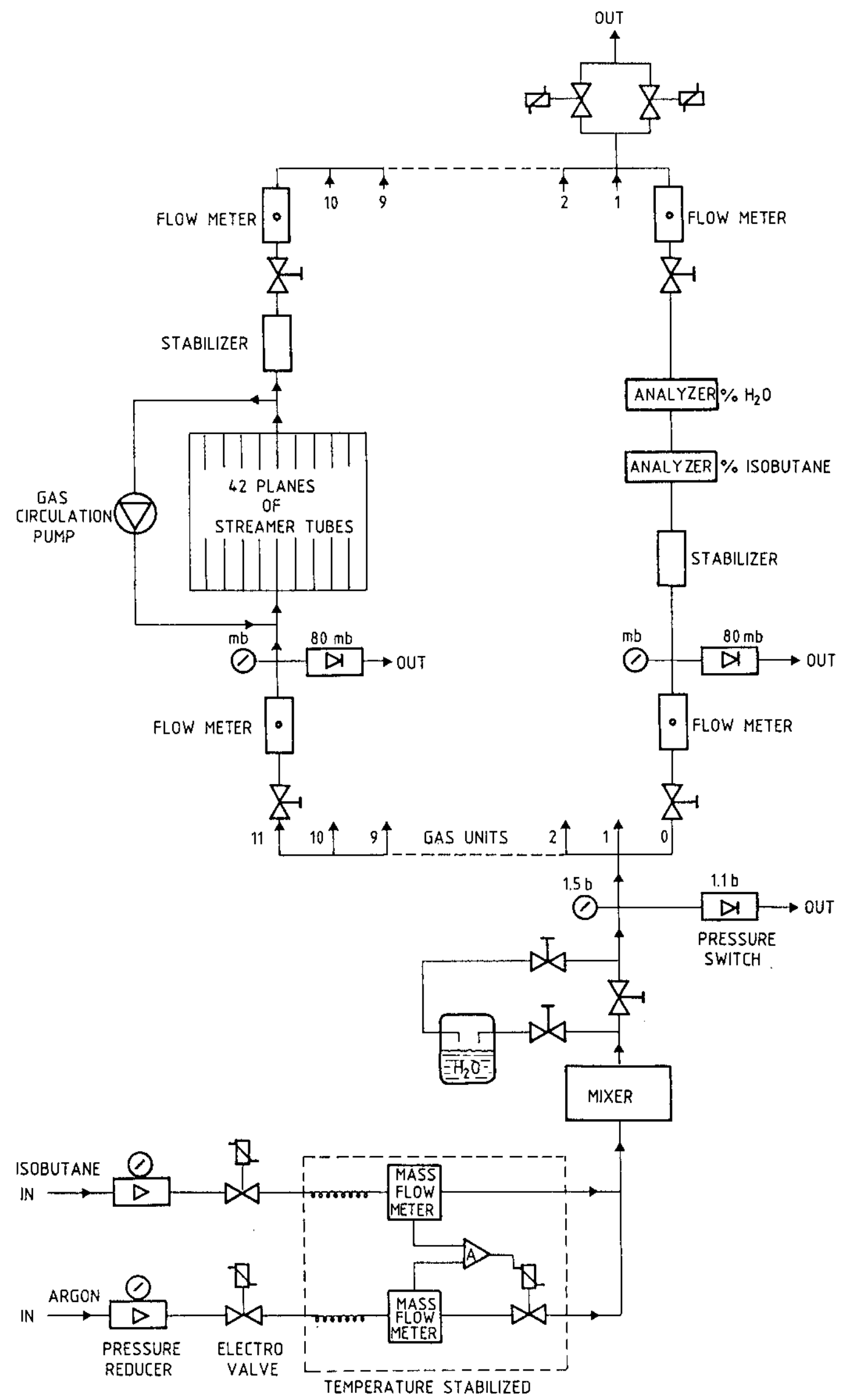

Fig. 3 


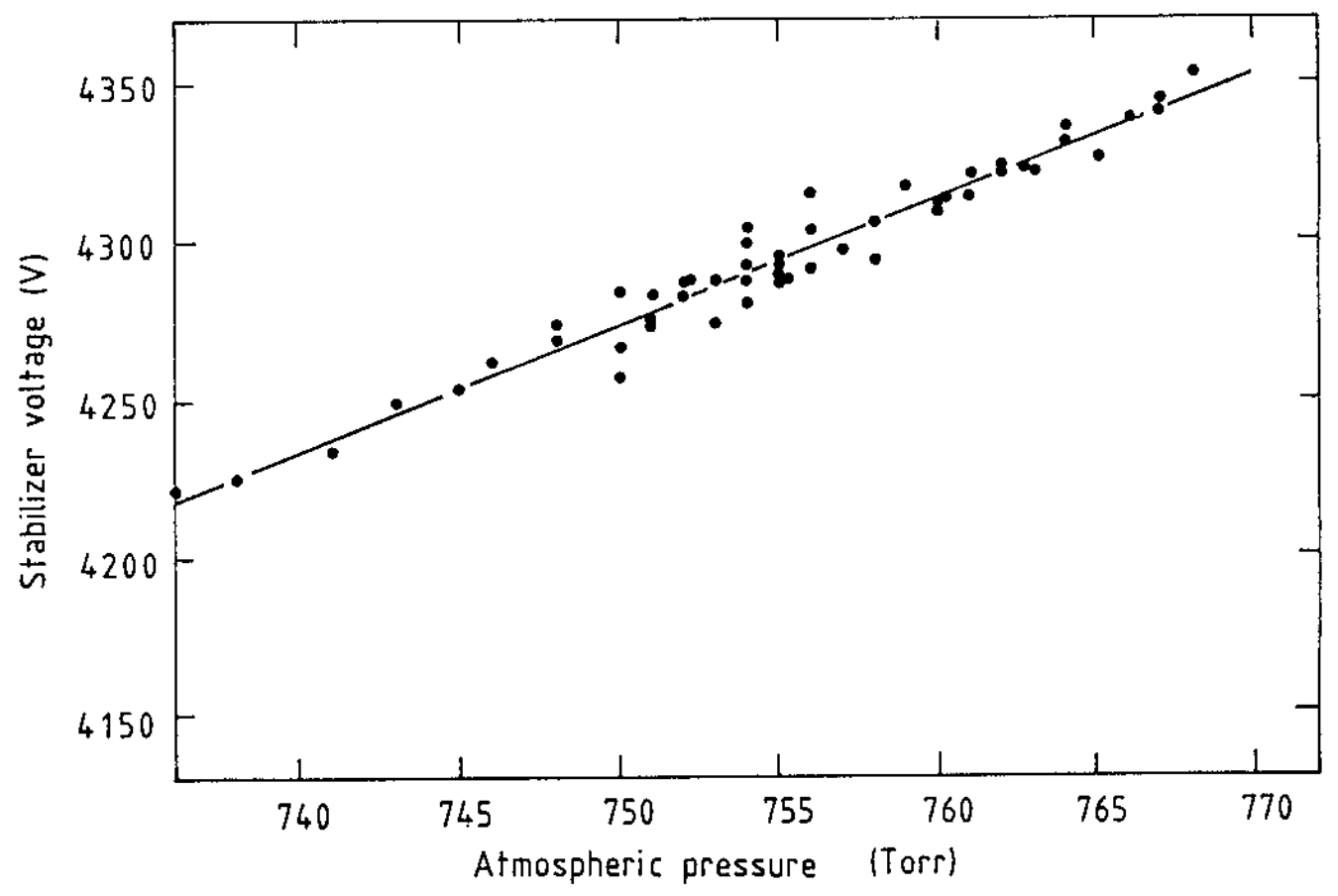

Fig. 4 

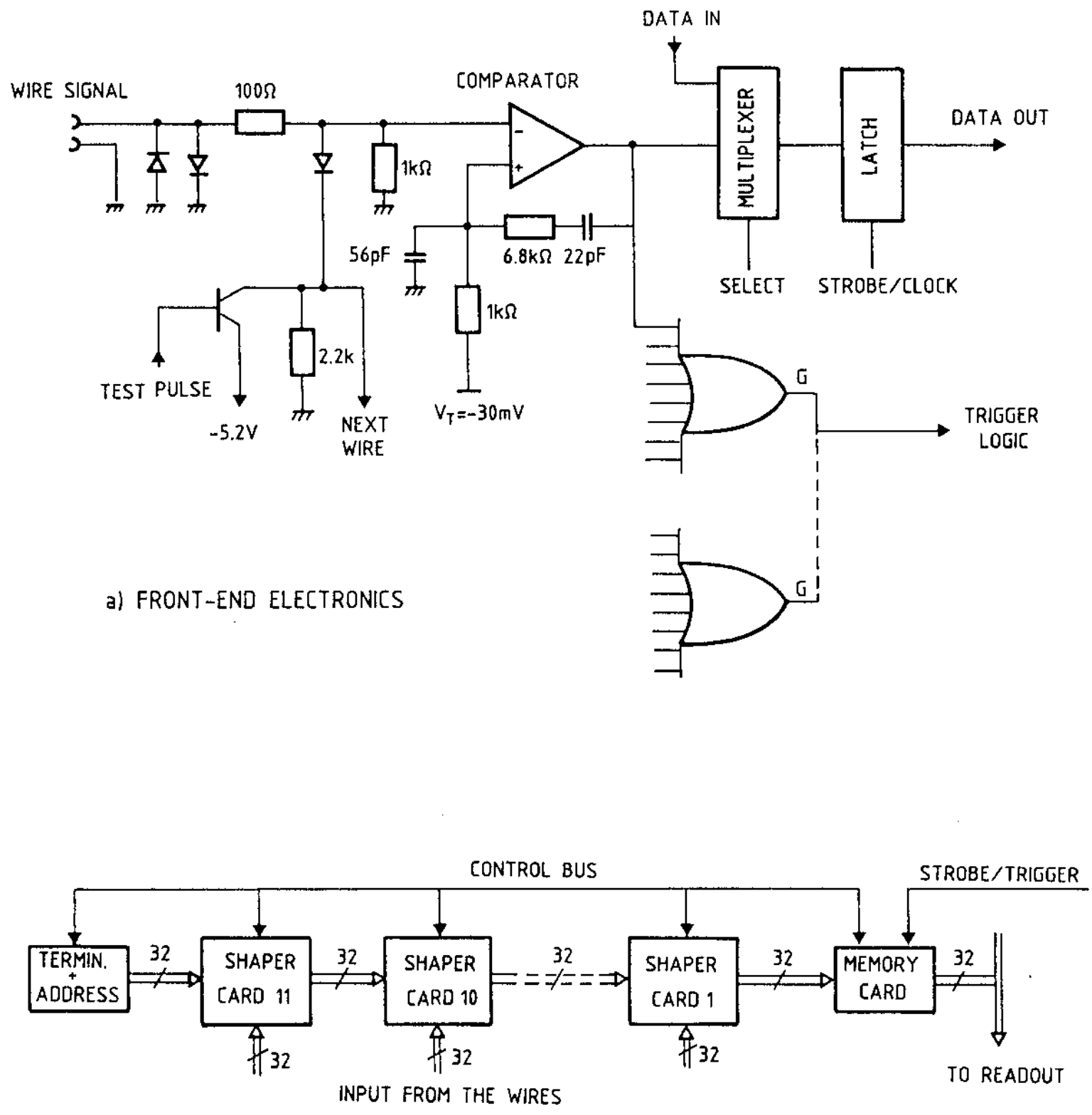

b) PLANE READOUT (EACH SHAPER (ARD READS 32 WIRES)

Fig. 5 


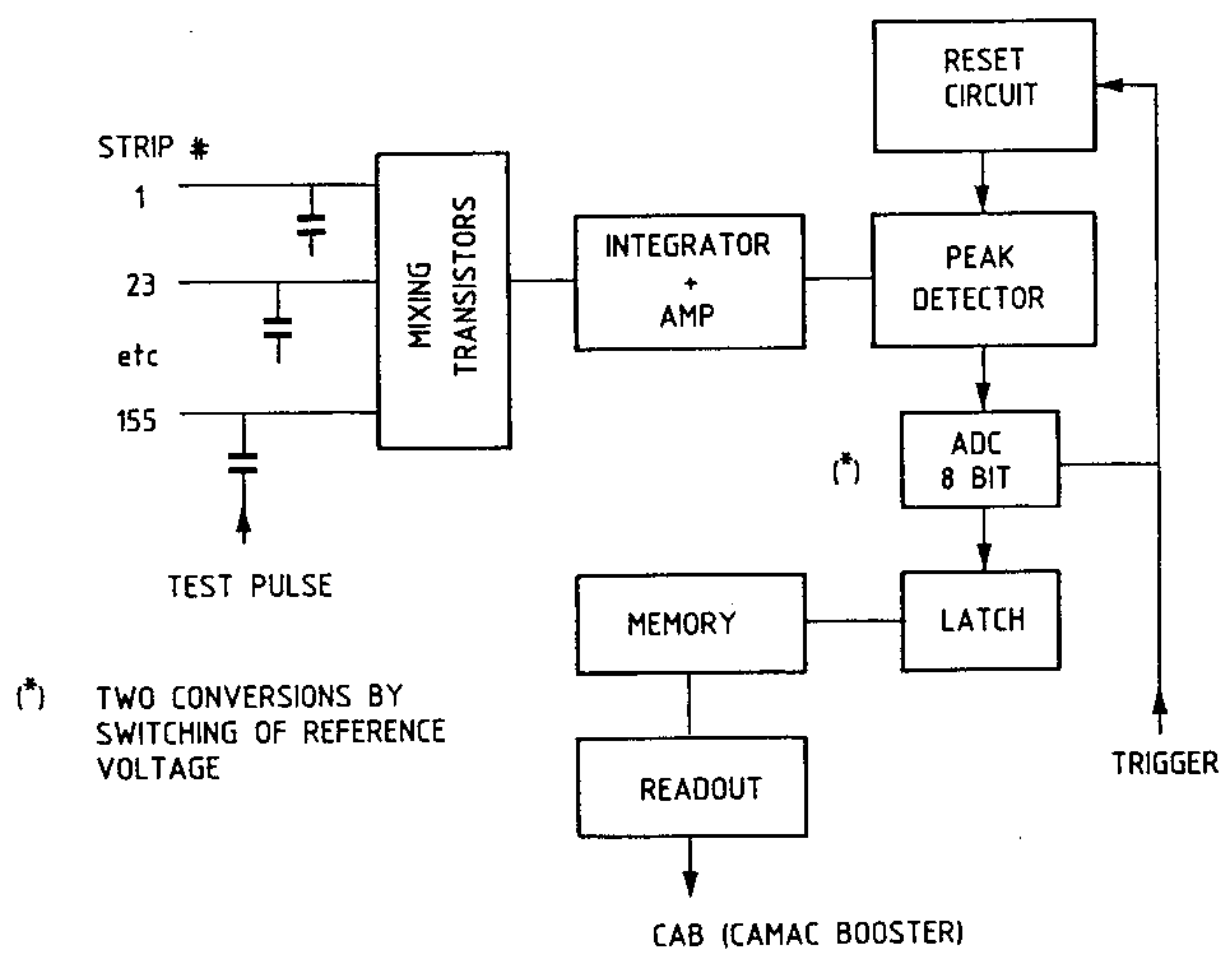

Fig. 6

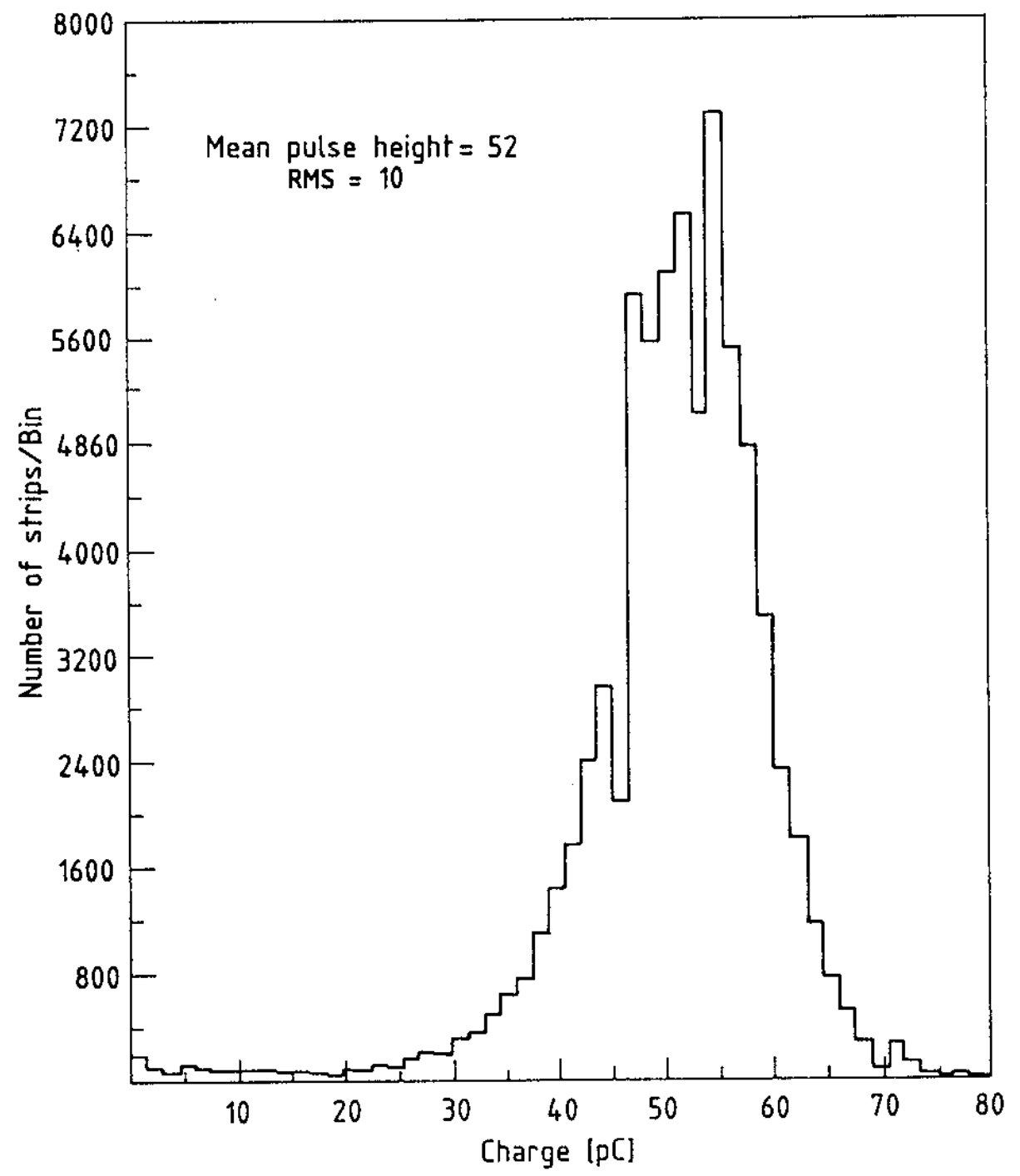

Fig. 7 


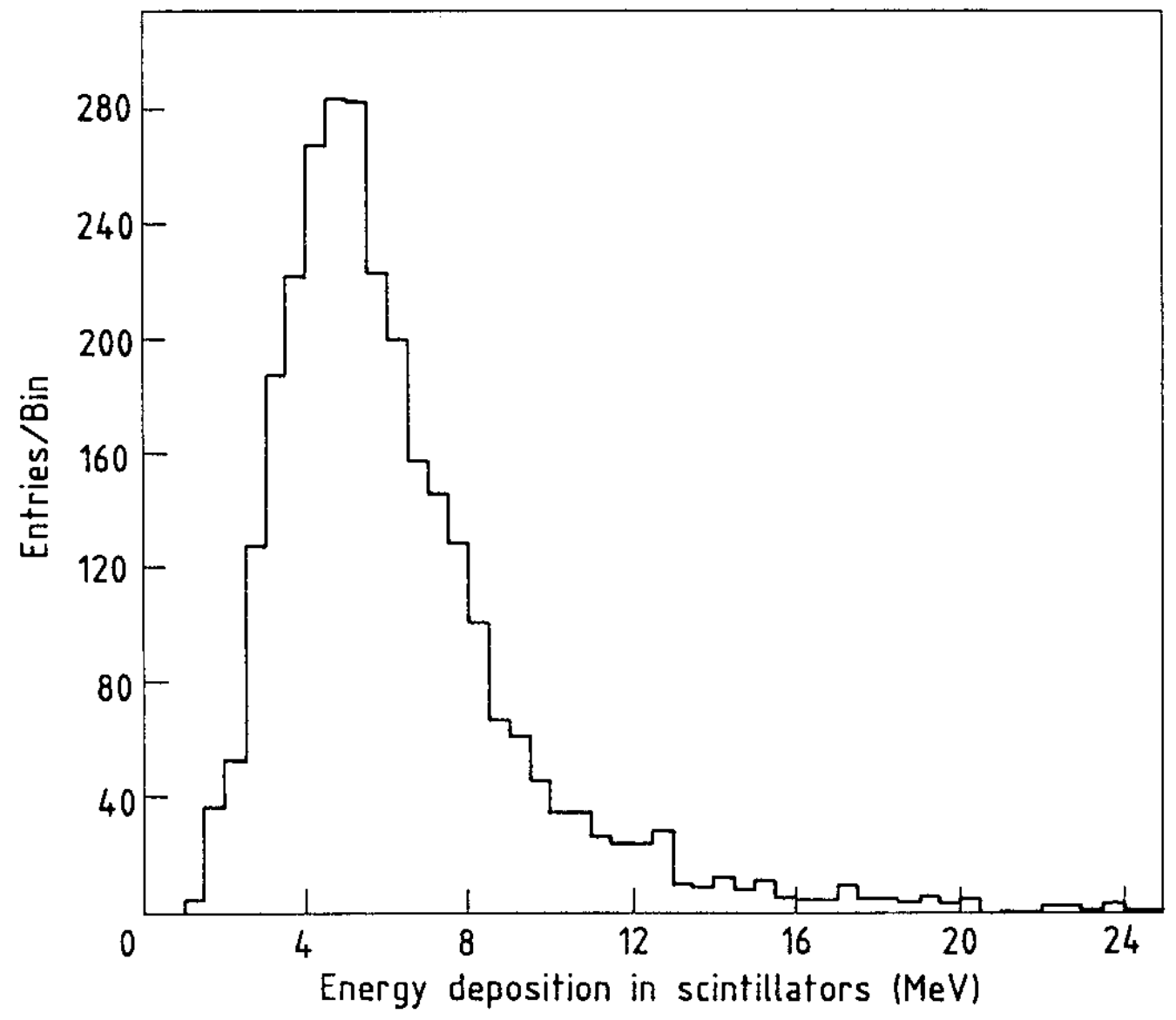

Fig. 8 


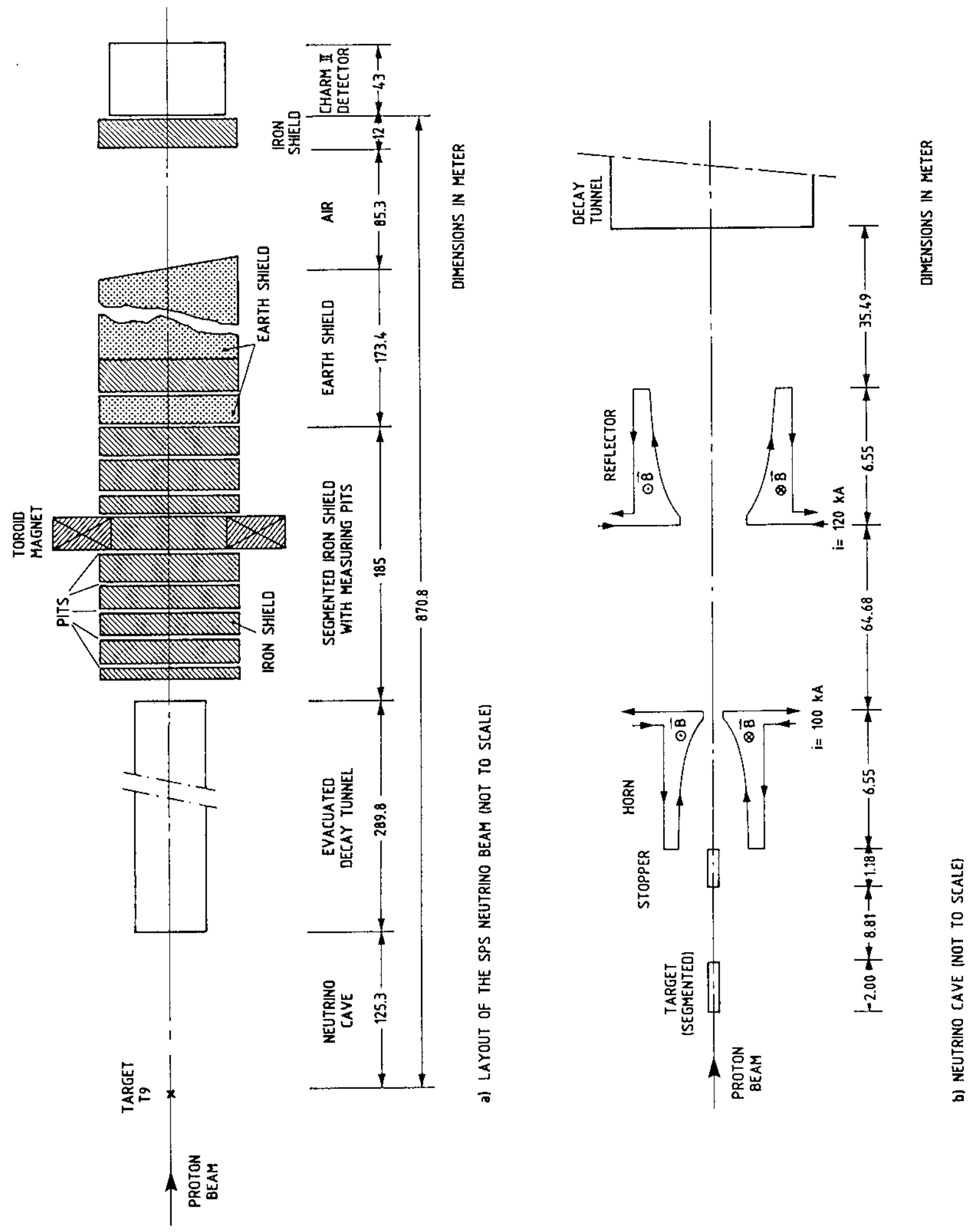




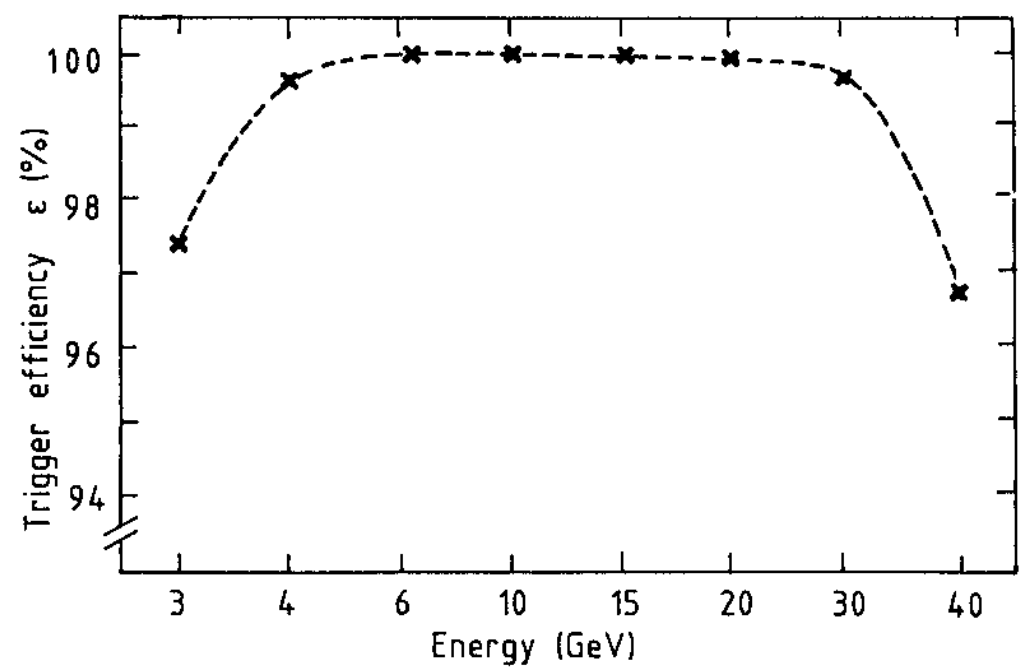

Fig. 10

a)

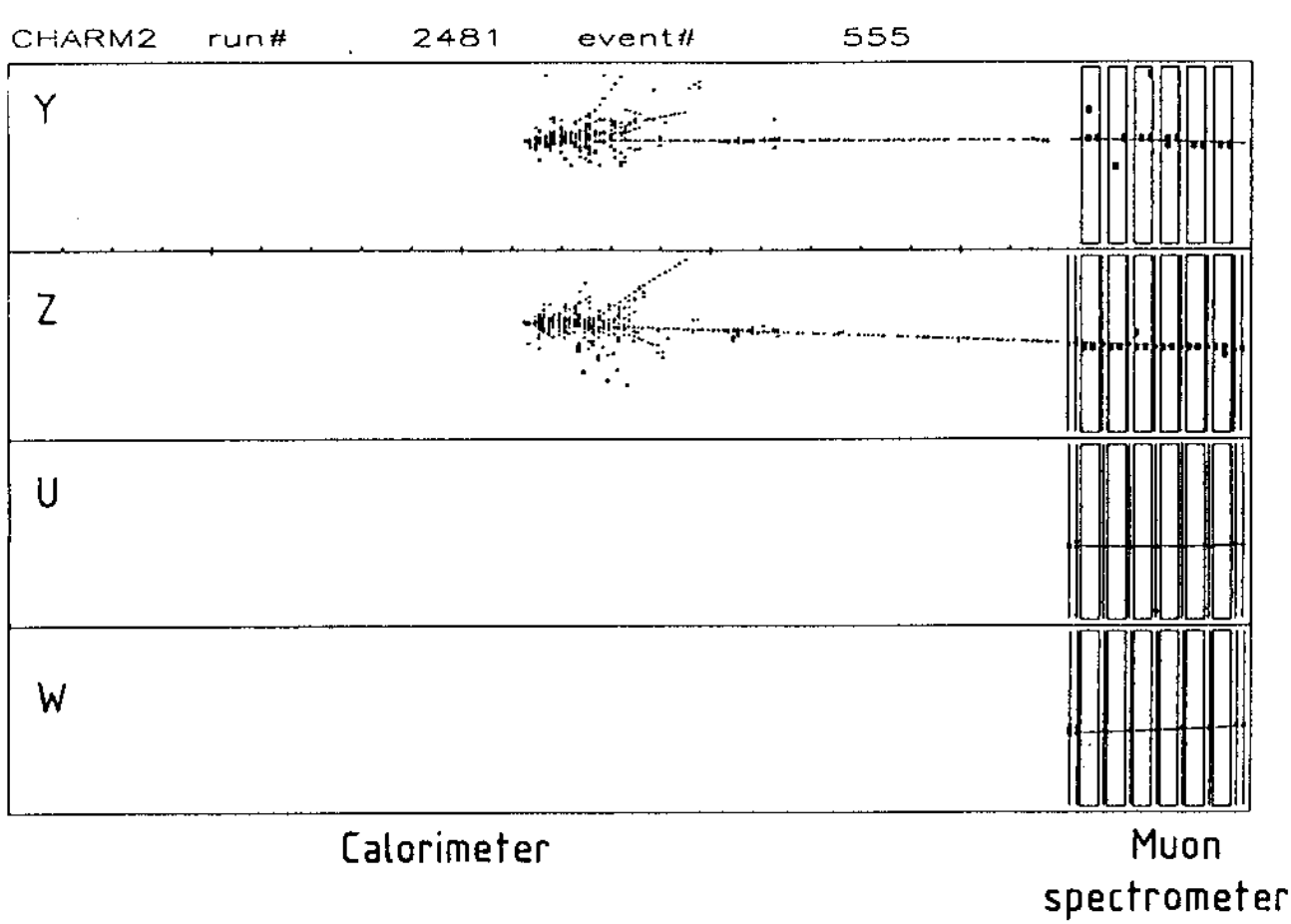

b) CHARMZ run\#

845 event\#

2797

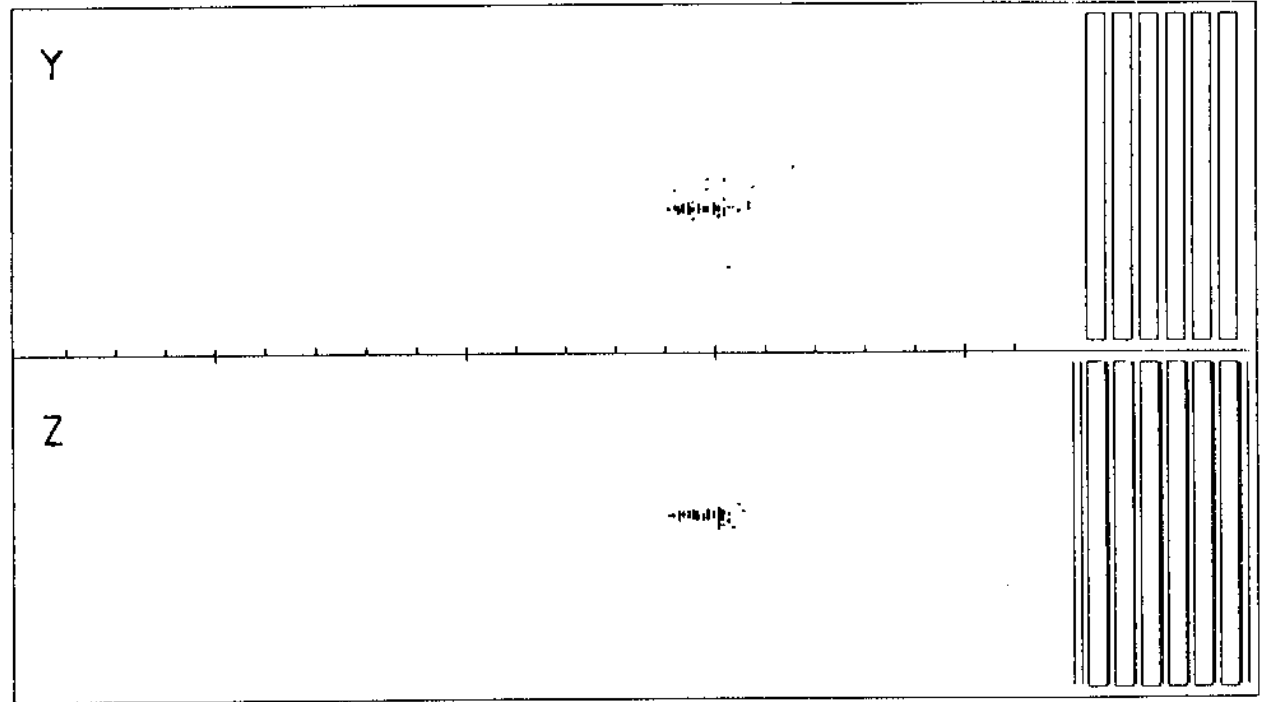

Fig. 11 

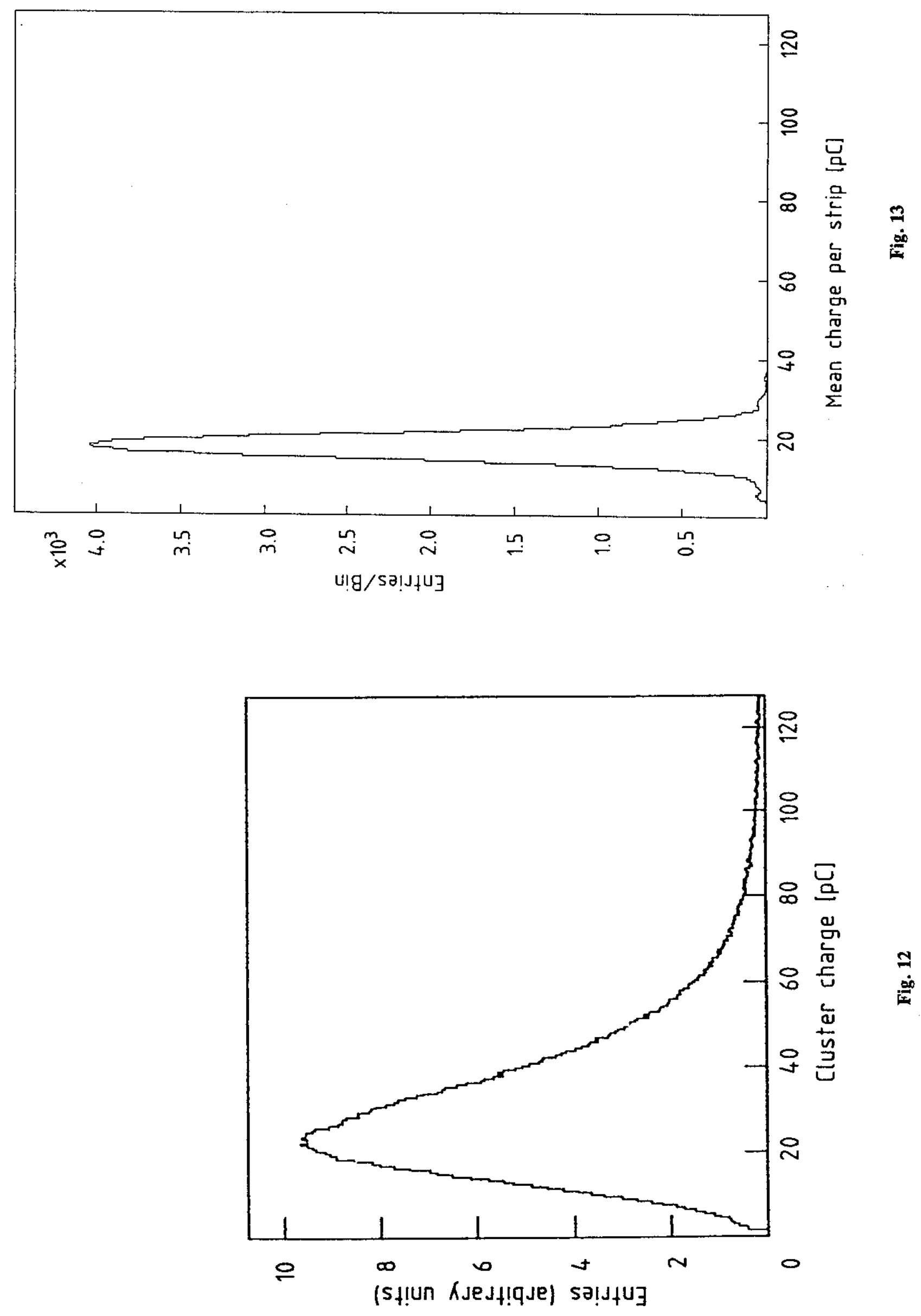


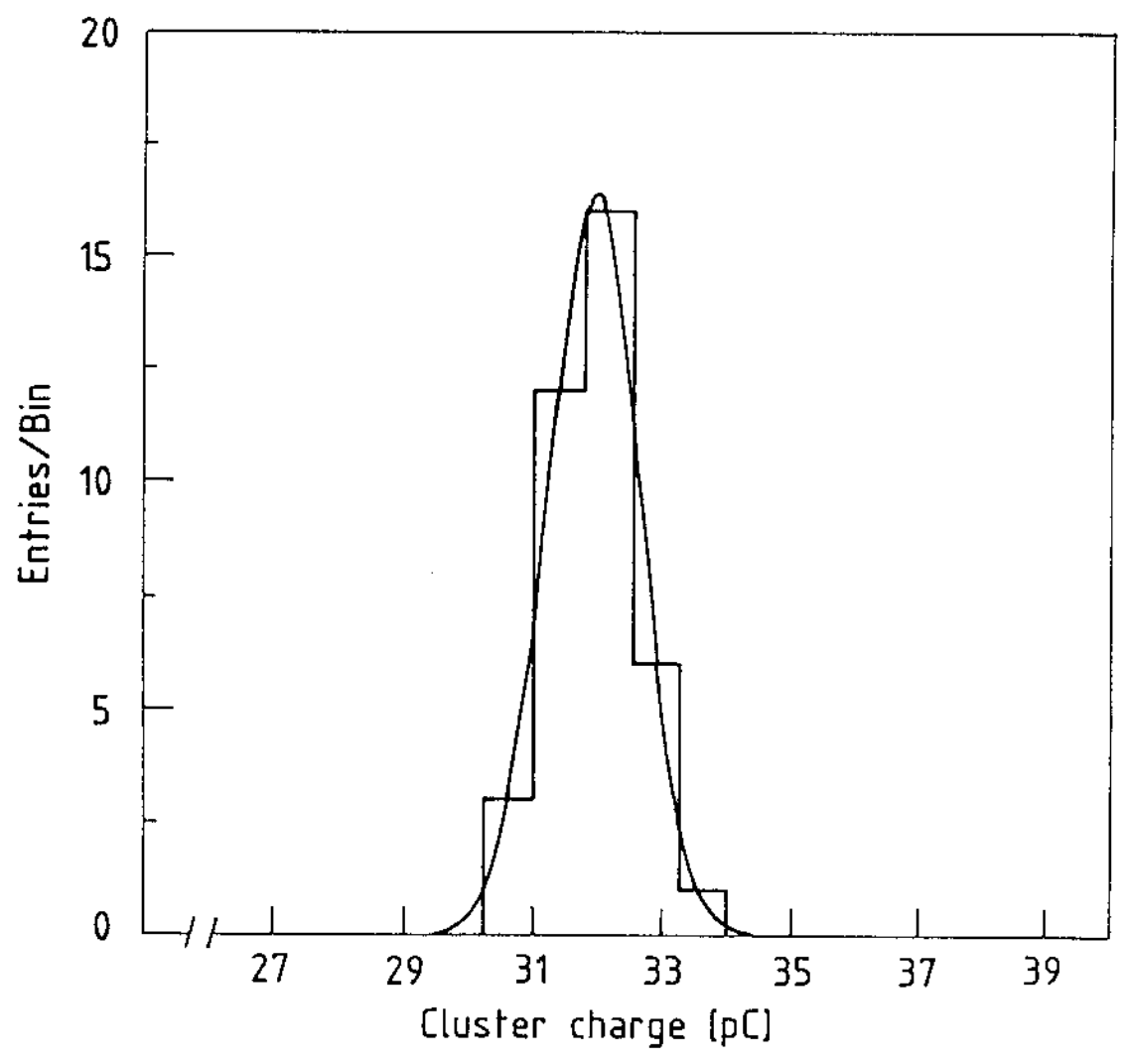

Fig. 14

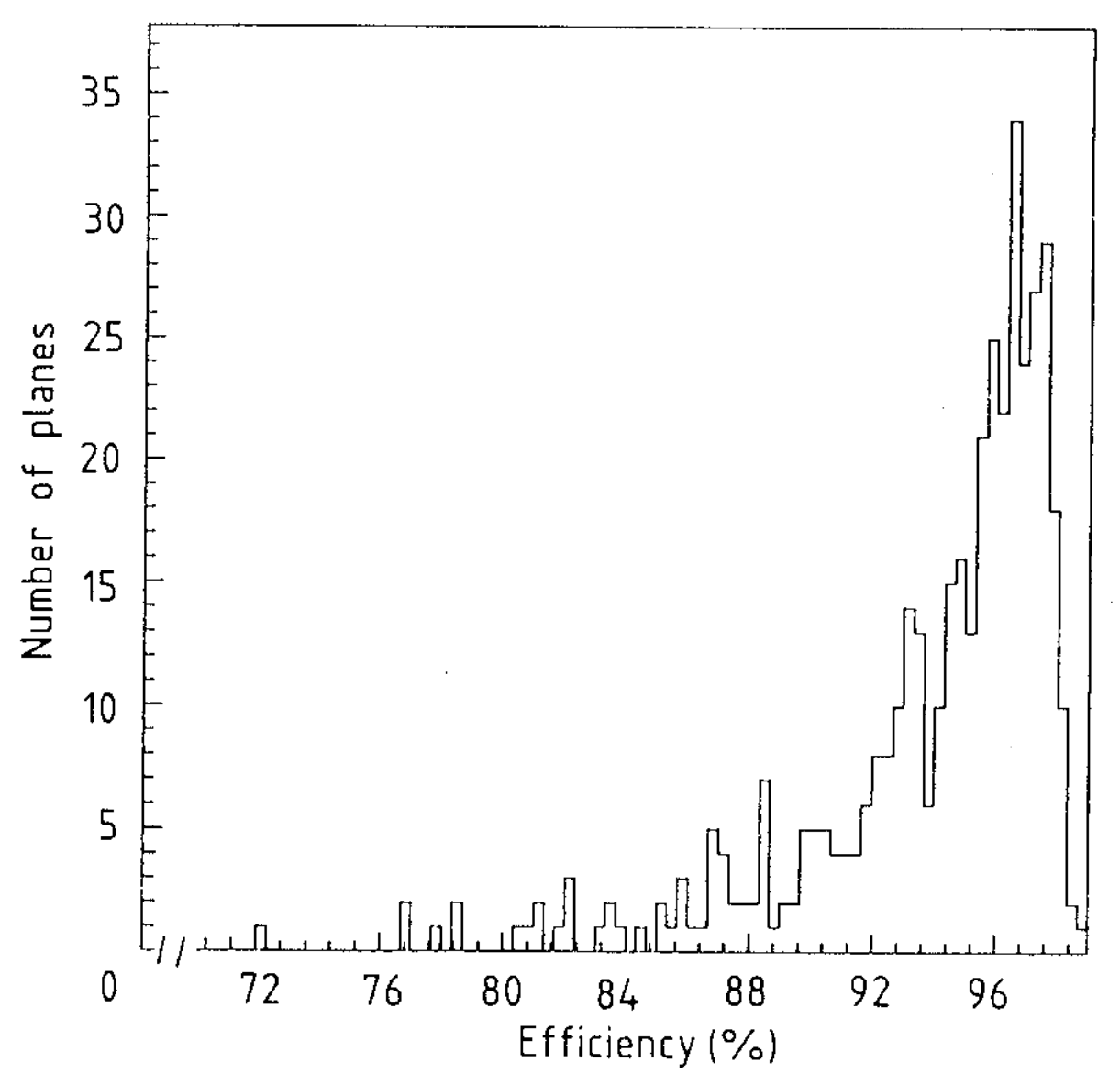

Fig. 15 


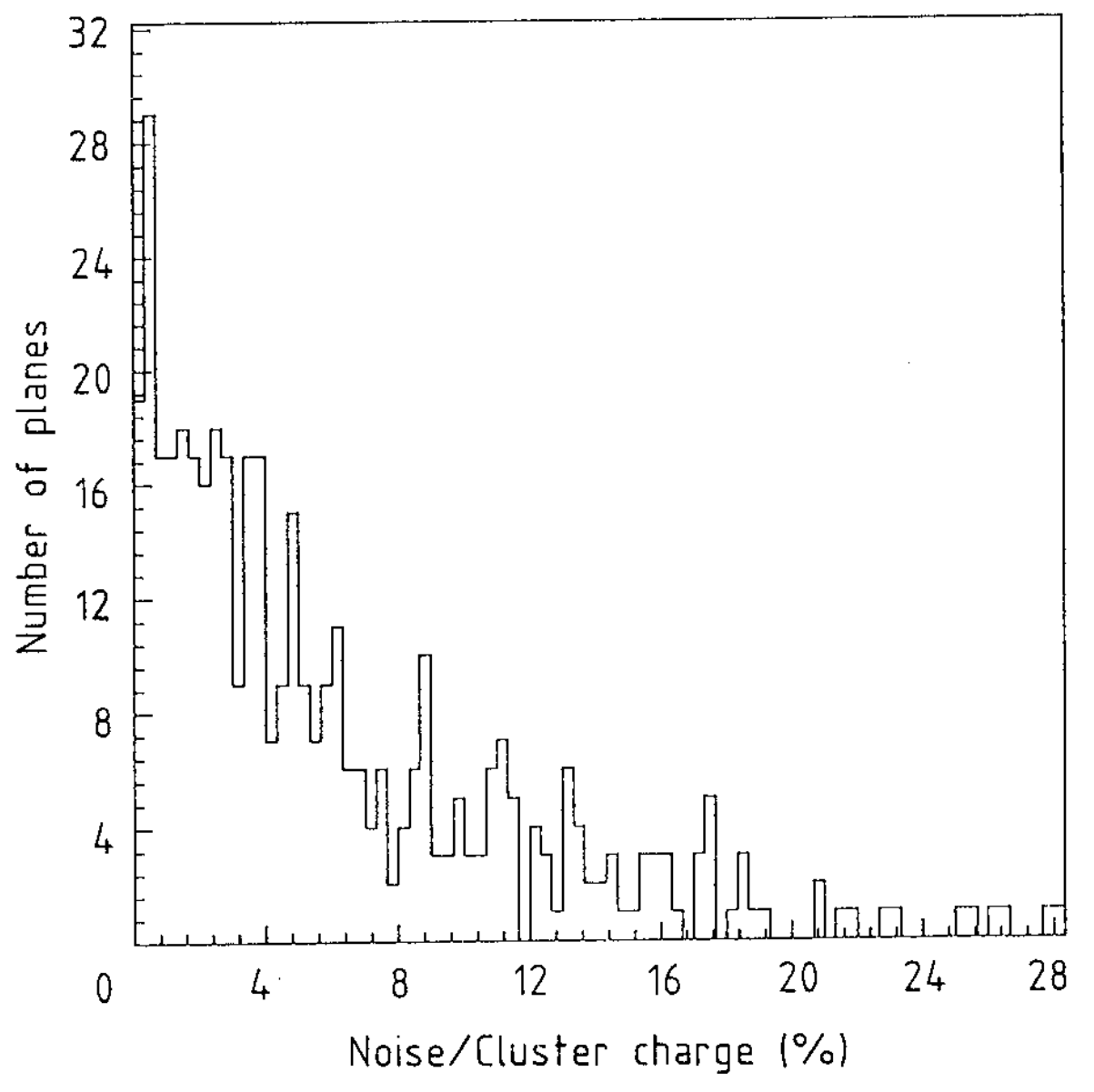

Fig. 16 